\title{
Actionable druggable genome-wide Mendelian randomization identifies repurposing opportunities for COVID-19
}

\author{
Liam Gaziano, ${ }^{1,2}$, Claudia Giambartolomei ${ }^{3,4}$, Alexandre C. Pereira ${ }^{5,6}$, Anna Gaulton ${ }^{7}$, \\ Daniel C. Posner (1) ', Sonja A. Swanson" , Yuk-Lam Ho', Sudha K. Iyengar9,10, Nicole M. Kosik ${ }^{1}{ }^{1}$, \\ Marijana Vujkovic $\circledR^{11,12}$, David R. Gagnon ${ }^{1,13}$, A. Patrícia Bento ${ }^{7}$, Inigo Barrio-Hernandez ${ }^{14}$, \\ Lars Rönnblom ${ }^{15}{ }^{15}$, Niklas Hagberg ${ }^{15}$, Christian Lundtoft ${ }^{15}{ }^{15}$, Claudia Langenberg ${ }^{16,17}$, \\ Maik Pietzner ${ }^{17}$, Dennis Valentine ${ }^{18,19}$, Stefano Gustincich $\mathbb{1}^{3}$, Gian Gaetano Tartaglia $\mathbb{1}^{3}$, \\ Elias Allara $\mathbb{1}^{2}$, Praveen Surendran 2,20,21,22, Stephen Burgess ${ }^{2,23}$, Jing Hua Zhao' ${ }^{2}$, James E. Peters $\mathbb{B}^{21,24}$, \\ Bram P. Prins ${ }^{2,21}$, Emanuele Di Angelantonio 2,20,21,25,26, Poornima Devineni', Yunling Shi', \\ Kristine E. Lynch ${ }^{27,28}$, Scott L. DuVall27,28, Helene Garcon', Lauren O. Thomann', Jin J. Zhou ${ }^{29,30,}$ \\ Bryan R. Gorman', Jennifer E. Huffman ${ }^{31}{ }^{31}$, Christopher J. O'Donnell ${ }^{32}{ }^{32,33}$, Philip S. Tsao ${ }^{34,35}$, \\ Jean C. Beckham ${ }^{36,37}$, Saiju Pyarajan1, Sumitra Muralidhar ${ }^{38}$, Grant D. Huang ${ }^{38}$, Rachel Ramoni ${ }^{38}$, \\ Pedro Beltrao (14, John Danesh 2,20,21,25,26, Adriana M. Hung ${ }^{39,40}$, Kyong-Mi Chang (12,41, \\ Yan V. Sun ${ }^{42,43}$, Jacob Joseph ${ }^{1,44}$, Andrew R. Leach7, Todd L. Edwards ${ }^{45,46}$, Kelly Cho ${ }^{1,47}$, \\ J. Michael Gaziano ${ }^{1,47}$, Adam S. Butterworth ${ }^{2,20,21,25,26 \bowtie \text {, Juan P. Casas }}{ }^{1,47 凶}$ and \\ VA Million Veteran Program COVID-19 Science Initiative*
}

Drug repurposing provides a rapid approach to meet the urgent need for therapeutics to address COVID-19. To identify therapeutic targets relevant to COVID-19, we conducted Mendelian randomization analyses, deriving genetic instruments based on transcriptomic and proteomic data for 1,263 actionable proteins that are targeted by approved drugs or in clinical phase of drug development. Using summary statistics from the Host Genetics Initiative and the Million Veteran Program, we studied 7,554 patients hospitalized with COVID-19 and $>1$ million controls. We found significant Mendelian randomization results for three proteins (ACE2, $P=1.6 \times 10^{-6}$; IFNAR2, $P=9.8 \times 10^{-11}$ and IL-10RB, $P=2.3 \times 10^{-14}$ ) using cis-expression quantitative trait loci genetic instruments that also had strong evidence for colocalization with COVID-19 hospitalization. To disentangle the shared expression quantitative trait loci signal for IL10RB and IFNAR2, we conducted phenome-wide association scans and pathway enrichment analysis, which suggested that IFNAR2 is more likely to play a role in COVID-19 hospitalization. Our findings prioritize trials of drugs targeting IFNAR2 and ACE2 for early management of COVID-19.

\footnotetext{
$\mathrm{T}$
} he global COVID-19 pandemic is responsible for substantial mortality, morbidity and economic hardship. Even with efficacious vaccines against the SARS-CoV-2 virus, it unknown how long it will take to achieve herd immunity, to what extent protection will diminish over time or if future mutations will enable SARS-CoV-2 to evade immune responses stimulated by current vaccines. Hence, there is a need to rapidly identify drugs that can minimize the burden of COVID-19. Although large randomized trials have begun to successfully identify drugs that can be repurposed to address COVID-19 (refs. ${ }^{1-3}$ ), most drugs evaluated so far have failed to show efficacy and have been largely confined to hospitalized or critically ill patients. It is a priority, therefore, to identify additional drugs that can be repurposed for early management in COVID-19.

Large-scale human genetic studies are now widely used to inform drug development programs. Drug-target disease pairs supported by human genetics have a greater odds of success in drug discovery pipelines ${ }^{4}$. For example, identification of variants in PCSK9 associated with lower risk of coronary disease led to the successful development of PCSK9 inhibitors, which are now licensed for prevention of cardiovascular events 5 . The value of human genetics for drug discovery and development has also been realized for infectious diseases. Human genetic studies showed that genetic variation in the CCR5 gene provides protection against infection by human immunodeficiency virus (HIV) type 1 . These findings were key for the development of Maraviroc, an antagonist of CCR5, approved by the US Food and Drug Administration (FDA) for the treatment of patients with HIV-1 (ref. ${ }^{6}$ ).

Genetic variants acting in 'cis' on druggable protein levels or gene expression that encode druggable proteins can provide powerful tools for informing therapeutic targeting, as they mimic the on-target 
(beneficial or harmful) effects observed by pharmacological modification ${ }^{7}$. Such Mendelian randomization (MR) analyses have been used to suggest repurposing opportunities for licensed drugs ${ }^{8}$. MR analysis that focuses on actionable druggable genes, defined as genes that encode the protein targets of drugs that are licensed or in the clinical phase of drug development, could therefore serve as a swift and robust strategy to identify drug-repurposing opportunities to prevent the complications and mortality due to COVID- 19 .

To identify further potential repurposing opportunities to inform trials of patients with COVID-19, we conducted large-scale $\mathrm{MR}$ and colocalization analyses using gene expression and soluble protein data for 1,263 actionable druggable genes that encode protein targets for approved drugs or drugs in clinical development. By combining transancestry genetic data from 7,554 hospitalized patients with COVID-19 and more than 1 million population-based controls from the COVID-19 Host Genetics Initiative ${ }^{9}$ (HGI) and the Million Veteran $\operatorname{Program}^{10}$ (MVP), we provide support for two therapeutic strategies.

\section{Results}

Overall analysis plan. Figure 1 describes the overall scheme of the analyses. First, we identified all proteins that are therapeutic targets of approved or clinical-stage drugs. Next, we selected conditionally independent genetic variants that act locally on plasma levels of these proteins or tissue-specific gene expression that encode these proteins. We proposed that these variants were instrumental variables and conduct two-sample MR analyses using a transancestry meta-analysis of 7,554 cases from MVP and publicly available data (HGI outcome B2 from release 4 v.1, downloaded 4 October 2020; Supplementary Table 1). Given that all MR analyses rely on several assumptions, some ${ }^{11}$ unverifiable, we conducted a multistage strategy to minimize confounding and biases. For MR results that passed our significance threshold after accounting for multiple testing, we performed colocalization to ensure MR results were not due to confounding by linkage disequilibrium (LD). Those with evidence of colocalization were investigated further using an independent proteomics platform (Olink). Finally, we conducted phenome-wide scans and pathway enrichment of relevant variants to reduce risks of horizontal pleiotropy and other biases due to MR violations as well as to understand potential biological mechanisms.

Actionable druggable proteins. Using data available in ChEMBL v.26, we identified 1,263 human proteins as 'actionable' (therapeutic targets of approved or clinical-stage drugs; Supplementary Table 2). Of these, we noted 700 proteins that are targets for drugs with potential relevance to COVID-19 from cell-based screening, registers of clinical trials against COVID-19 or approved immunomodulatory/anticoagulant drugs (given the clear role of these pathways in COVID-19 outcomes) or have biological evidence for the role of the protein in SARS-CoV-2 infection (Supplementary Table 3).

Genetic proposed instruments for actionable druggable proteins. Using GTEx v.8 ( ref. $^{12}$ ), we identified all conditionally independent expression quantitative trait loci (eQTLs) in 49 tissues that act in cis (within $1 \mathrm{Mb}$ on either side of the encoded gene), which covered 1,016 of the 1,263 druggable genes in at least one tissue (Supplementary Tables 2 and 4). We also selected cis-protein quantitative trait loci (pQTLs) for plasma proteins measured using the SomaScan platform in 3,301 participants of the INTERVAL study ${ }^{13}$ (Supplementary Table 5) and 10,708 Fenland cohort participants ${ }^{14}$ (Supplementary Table 6) that covered a total of 67 proteins. In total 1,021 proteins had genetic proposed instruments using either eQTLs or pQTLs and 62 had proposed instruments using both.

Mendelian randomization and colocalization. Using our (eQTL and $\mathrm{PQTL}$ ) proposed instruments, we performed two-sample MR

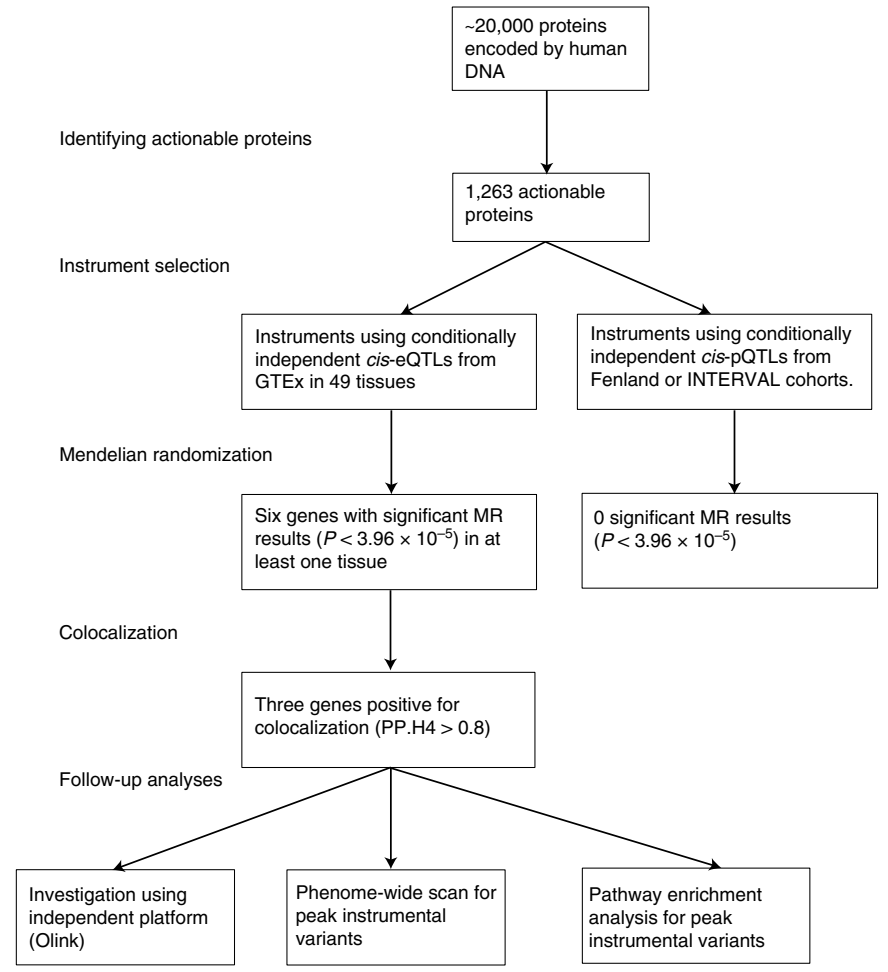

Fig. 1 | Outline of the analyses performed. Using multiple data sources, this study tested cis-pQTL and cis-eQTL proposed instruments for actionable druggable proteins against COVID-19 hospitalization summary statistics meta-analyzed from the HGI and the MVP. Significant MR associations that also showed evidence for colocalization were investigated further with an independent platform (Olink), phenome-wide scans of relevant variants and pathway enrichment.

on transancestry summary statistics for hospitalized patients with COVID-19 from MVP and HGI (Supplementary Table 1). Using GTEx cis-eQTLs as proposed instruments, we found significant $\left(P<3.96 \times 10^{-5}, 0.05\right.$ Bonferroni-corrected for 1,263 actionable proteins) MR results for six genes (IL10RB, CCR1, IFNAR2, PDE4A, $A C E 2$ and CCR5) in at least one tissue (MR results with $P<3.96 \times 10^{-5}$ shown in Table 1 and full MR results in Supplementary Table 7) and four additional genes (CA5B, CA9, NSTN and SLC9A3) with suggestive MR results $\left(P<5.00 \times 10^{-4}\right.$ and $P>3.96 \times 10^{-5}$; Fig. 2 and Supplementary Table 7). No proposed instruments involving cis-pQTLs reached our suggestive threshold in any of the analyses (Supplementary Tables 8 and 9). For three significant genes (IL10RB, IFNAR2 and ACE2) there was strong evidence of colocalization (posterior probability of shared causal variant across two traits, hypothesis $4(\mathrm{PP} . \mathrm{H} 4)>0.80)$ between at least one proposed instrumental variant and our transancestry meta-analysis of COVID-19 hospitalization (Table 1). The $\beta$-coefficients of MR estimates for ACE2 were positive in all tissues (Table 1), meaning higher ACE2 expression is associated with higher risk of COVID-19 hospitalization. MR $\beta$-coefficients for IFNAR2 and IL1ORB were negative and positive, respectively in all tissues except one for each gene (skeletal muscle for IFNAR2; cultured fibroblasts for IL1ORB; Table 1).

IL10RB and IFNAR2. Interferon (IFN)- $\alpha$ receptor 2 (IFNAR2) and interleukin (IL)-10 receptor- $\beta$ (IL-10RB) both act as receptors for IFNs. IFNAR2 forms a complex with IFNAR1, which together act as a receptor for type I IFN (IFN- $\alpha, \beta, \omega, \kappa, \varepsilon)$, whereas IL-10RB acts as a receptor for type III IFN (IFN- $\lambda$ ) when complexed with IFN- $\lambda$ receptor 1 (IFNLR 1$)^{15}$ or IL-10 when complexed with IL-10RA. IL-10RB and IFNAR2 are encoded by adjacent genes and some cis-eQTLs 
Table 1 | Significant $\left(P<3.96 \times 10^{-5}\right)$ MR results

\begin{tabular}{|c|c|c|c|c|c|c|c|}
\hline Gene & Tissue & $\boldsymbol{\beta}$ & s.e. & $P$ value & $\boldsymbol{P}_{\text {het }}$ & Variants in instrument & Colocalization \\
\hline IL10RB & Muscle skeletal & 0.5078 & 0.0665 & $2.31 \times 10^{-14}$ & 0.9732 & rs2300370, rs2834167 & $0.98,<0.01$ \\
\hline IL10RB & Nerve tibial & 0.2859 & 0.0384 & $9.76 \times 10^{-14}$ & 0.0052 & rs13050728, rs2834167, rs2266590 & $\begin{array}{l}0.98,<0.01 \\
<0.01\end{array}$ \\
\hline CCR1 & Cells cultured fibroblasts & 0.4449 & 0.0612 & $3.60 \times 10^{-13}$ & NA & rs13095940 & $<0.01$ \\
\hline IL10RB & $\begin{array}{l}\text { Brain nucleus accumbens } \\
\text { basal ganglia }\end{array}$ & 0.2541 & 0.0363 & $2.58 \times 10^{-12}$ & 0.0019 & rs2834167, rs17860115 & $0.75,0.98$ \\
\hline IL1ORB & $\begin{array}{l}\text { Brain caudate basal } \\
\text { ganglia }\end{array}$ & 0.2635 & 0.0398 & $3.61 \times 10^{-11}$ & 0.0003 & rs2834167, rs1051393 & $0.01,0.97$ \\
\hline IFNAR2 & Muscle skeletal & 0.5881 & 0.0909 & $9.75 \times 10^{-11}$ & NA & rs2300370 & 0.98 \\
\hline IL1ORB & $\begin{array}{l}\text { Brain cerebellar } \\
\text { hemisphere }\end{array}$ & 0.1405 & 0.0229 & $8.22 \times 10^{-10}$ & 0.0389 & rs2834167, rs2236758 & $0.01,0.95$ \\
\hline IL10RB & Breast mammary tissue & 0.6490 & 0.1079 & $1.82 \times 10^{-9}$ & NA & rs12053666 & 0.95 \\
\hline IL10RB & Brain frontal cortex BA9 & 0.4667 & 0.0790 & $3.55 \times 10^{-9}$ & 0.0366 & rs2834167, rs1131668 & $0.14,0.97$ \\
\hline IL10RB & Brain cortex & 0.1929 & 0.0328 & $3.99 \times 10^{-9}$ & 0.0354 & rs2834167, rs1131668 & $0.02,0.96$ \\
\hline CCR1 & $\begin{array}{l}\text { Esophagus } \\
\text { gastroesophageal } \\
\text { junction }\end{array}$ & 0.1776 & 0.0302 & $4.11 \times 10^{-9}$ & NA & rs13059906 & 0.05 \\
\hline IL1ORB & Brain cerebellum & 0.1147 & 0.0197 & $5.82 \times 10^{-9}$ & 0.0239 & rs2834167, rs1131668 & $<0.01,0.96$ \\
\hline CCR1 & Esophagus mucosa & 0.4338 & 0.0751 & $7.60 \times 10^{-9}$ & NA & rs34059564 & $<0.01$ \\
\hline IFNAR2 & Esophagus mucosa & -0.4883 & 0.0865 & $1.63 \times 10^{-8}$ & NA & rs11911133 & 0.92 \\
\hline PDE4A & Artery aorta & -0.5420 & 0.0965 & $1.98 \times 10^{-8}$ & 0.0202 & rs370630099, rs45524632 & $0.41,0.61$ \\
\hline IL10RB & Testis & 0.7104 & 0.1364 & $1.92 \times 10^{-7}$ & NA & rs2284550 & 0.11 \\
\hline IFNAR2 & $\begin{array}{l}\text { Skin not sun-exposed, } \\
\text { suprapubic }\end{array}$ & -0.3360 & 0.0671 & $5.46 \times 10^{-7}$ & NA & rs 8127500 & $<0.01$ \\
\hline IFNAR2 & Pancreas & -0.4708 & 0.0957 & $8.63 \times 10^{-7}$ & NA & rs1476415 & 0.06 \\
\hline ACE2 & Brain frontal cortex BA9 & 0.1121 & 0.0233 & $1.56 \times 10^{-6}$ & NA & rs4830976 & 0.95 \\
\hline IFNAR2 & Cells cultured fibroblasts & -0.3893 & 0.0819 & $1.98 \times 10^{-6}$ & NA & rs1131668 & 0.92 \\
\hline IL1ORB & Cells cultured fibroblasts & -0.5197 & 0.1093 & $1.98 \times 10^{-6}$ & NA & rs1131668 & 0.96 \\
\hline CCR5 & Lung & -0.5868 & 0.1272 & $3.99 \times 10^{-6}$ & NA & rs12639314 & 0.02 \\
\hline IL10RB & $\begin{array}{l}\text { Esophagus } \\
\text { gastroesophageal junction }\end{array}$ & 0.4678 & 0.1052 & $8.80 \times 10^{-6}$ & NA & rs56079299 & 0.96 \\
\hline
\end{tabular}

Significant MR results, $P<3.96 \times 10^{-5}$ ( 0.05 Bonferroni-corrected for 1,263 actionable druggable genes). MR estimates were calculated using inverse-variance weighting and fixed effects for instruments that contained more than one variant and Wald ratio for instruments with one variant. All results used cis-eQTL instruments and no results using cis-pQTL instruments yielded results $P<3.96 \times 10^{-5} . P_{\text {het }}$ refers to the heterogeneity $P$ value across individual-variant MR estimates within a genetic instrument calculated using the Cochrane $Q$ method, therefore instruments containing one variant were not tested for heterogeneity. A positive $\beta$ estimate indicates that more gene expression is associated with higher risk of COVID-19 hospitalization. 'Colocalization' indicates PP.H4 between eQTLs and COVID-19 hospitalization. For example, for IL1ORB in skeletal muscle, the primary GWAS with rs 2300370 as the peak cis-eQTL colocalizes with COVID-19 hospitalization at PP.H4 $=0.98$ and the secondary GWAS (after adjusting for rs2300370) with rs2834167 as the peak cis-eQTL does not colocalize with COVID-19 hospitalization (PP.H4<0.01). NA, not available; s.e., standard error.

for IL10RB are also cis-eQTLs for IFNAR2 (Supplementary Table 10 and Fig. 3), making it difficult to determine which gene may be responsible for the association with COVID-19 and requiring further investigation.

All significant MR results for IFNAR2/IL10RB that colocalized with COVID-19 hospitalization contained one of nine strongly correlated ( $r^{2}>0.75$ in 1000 Genomes Project European (1000G EUR) ancestry participants) variants (rs11911133, rs1051393, rs2300370, rs56079299, rs17860115, rs13050728, rs2236758, rs12053666 and rs1131668), which are cis-eQTLs for IL10RB in 11 tissues and for IFNAR2 in 4 tissues (Supplementary Table 11). Within this LD block (hereafter rs13050728-LD block), rs13050728 is the eQTL most strongly associated with COVID-19 hospitalization (per T-allele odds ratio $=1.17 ; 95 \% \mathrm{CI}=1.12-1.23 ; \quad P=1.88 \times 10^{-12}$; Supplementary Table 10). Variants outside the rs13050728-LD block were not strongly associated with COVID-19 hospitalization (Fig. 3).

pQTLs for IL10RB. Using stepwise conditional analysis on Olink measurements of plasma IL-10RB, we identified two cis-pQTLs, rs2266590 $\left(P=1.04 \times 10^{-136}\right)$ and $\operatorname{rs} 2239573\left(P=2.66 \times 10^{-19}\right)$, which explained $5.4 \%$ and $1.2 \%$, respectively of the variance in plasma IL-10RB. rs2266590 was also an eQTL for IL10RB in three tissues and IFNAR2 in one tissue, while rs2239573 was also an eQTL for IL10RB in two tissues (Supplementary Table 11). rs2266590 and rs2239573 lie in intron 5 and 1, respectively of the IL10RB gene and are located in separate regions of high epigenetic modification (h3k27ac marking), indicating enhancer regions (Fig. 3). rs2266590 and rs2239573 were not associated with COVID-19 hospitalization ( $P=0.85$ for rs2266590, $P=0.66$ for rs2239573; Extended Data Fig. 1$)$ and MR using these two cis-pQTLs yields a null result $(P=0.74)$.

A third $c i s$-pQTL (rs2834167, $P=1.1 \times 10^{-8}$ ) for plasma IL-10RB measured on the SomaScan platform was previously identified in 3,200 Icelanders over the age of 65 years $^{16}$. rs2834167 is a missense variant (Lys $>$ Glu) and is not correlated with either of the cis-pQTLs for plasma IL-10RB measured by Olink $\left(r^{2}=0.01\right.$ for $r s 2266590$, $r^{2}=0.03$ for $r 2239573$ in 1000G EUR). Although rs2834167 was associated with IL10RB expression in 18 tissues, it was not associated with IFNAR2 expression in any tissue (Supplementary Table 11). 


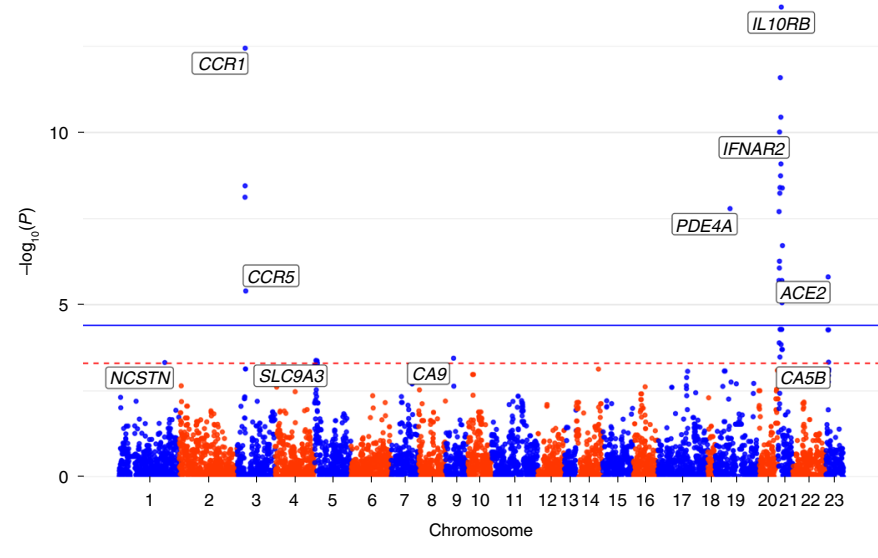

Fig. 2 | Manhattan plot of results from actionable druggable genome-wide MR analysis. MR estimates were calculated using inverse-variance weighting and fixed effects for instruments that contained more than one variant and Wald ratio for instruments with one variant. Blue solid line indicates $P$ value threshold for significance $\left(P<3.96 \times 10^{-5}, 0.05\right.$ Bonferroni-corrected for 1,263 actionable druggable genes) and red dashed line indicates a suggestive $\left(P<5.00 \times 10^{-4}\right)$ threshold. Genes are labeled by their strongest MR association. For example, the association for IL1ORB is strongest using cis-eQTL proposed instruments derived in skeletal muscle tissue $\left(P=2.31 \times 10^{-14}\right)$, which is the point labeled. Results are plotted by gene start position. All MR results with a $P$ value $<5.00 \times 10^{-4}$ used the GTEx cis-eQTLs as proposed instruments.

The A allele at rs2834167, which is associated with lower IL10RB gene expression but higher plasma IL-10RB, was inversely associated with COVID-19 (per-A-allele $\mathrm{OR}=0.91 ; 95 \% \mathrm{CI}=0.87-0.95$; $\left.P=5.3 \times 10^{-5}\right)$. Because Emilsson et al. ${ }^{16}$ did not report full summary statistics we could not perform colocalization between this pQTL and COVID-19 hospitalization. However, rs2834167 as an eQTL does not colocalize (PP.H4 < 0.8) with COVID-19 in any tissue (Table 1). These three cis-pQTLs, while possibly functional variants altering plasma IL-10RB levels, suggest that the plasma IL-10RB levels are not likely the mediator of the association between this locus and COVID-19 hospitalization. IFNAR2 was not measured on the SomaScan or Olink platforms.

Phenome-wide scan of rs13050728. To identify other phenotypes associated with rs13050728, we performed a phenome-wide scan of genome-wide association study (GWAS) for proteins measured by Olink and SomaLogic platforms in INTERVAL participants (Methods) and publicly available data on PhenoScanner ${ }^{17}$ and GTEx. rs 13050728 was associated with tryptase- $\gamma 1$ (TPSG1, $P=1.5 \times 10^{-5}$ ) and vascular endothelial growth factor 2 (VEGFR2, $P=2.6 \times 10^{-5}$; Supplementary Table 12) and both showed strong evidence of colocalization with COVID-19 hospitalization (PP.H4 $=0.96$ for VEGFR2, PP.H4 $=0.96$ for TPSG1; Fig. 4). The C allele at rs 13050728 associated with higher IFNAR2 expression in all tissues (except skeletal muscle), lower risk of COVID-19 hospitalization and lower levels of plasma VEGFR2 and TPSG1 (Supplementary Table 12). This mimics agonistic effects of IFNAR2 through recombinant type I IFNs, which are known to have an anti-angiogenic effect, at least in part through reduced VEGF/VEGFR2 signaling ${ }^{18,19}$ and decrease tryptase levels in a phase 2 trial using recombinant type I IFN in patients with mastocytosis ${ }^{20}$, a condition that causes proliferation of mast cells. rs 13050728 was not associated $\left(P<3.96 \times 10^{-5}\right.$ Bonferroni-corrected $P$ value) with any phenotype beyond plasma VEGFR2 and TPSG1 and gene expression of IFNAR2 and IL10RB (Supplementary Table 12), indicating that this variant is unlikely to exhibit widespread horizontal pleiotropy. Also, the chances of substantial bias due to MR violations is $l^{21} \mathrm{w}^{21}$ because the variant is not strongly associated with other risk factors that could alter the likelihood of SARS-CoV-2 testing or hospitalization of patients with COVID-19.

Pathway enrichment analysis of rs13050728. Using information from all GTEx v.8 tissues we identified 476 genes whose expression levels were associated with rs 13050728 at a nominal significance level $(P<0.05)$. Taking into consideration an adjusted $P$ value for multiple testing within the WikiPathway corpus, only two biological pathways were significantly associated among all 624 pathways present in this database: host-pathogen interaction of human corona viruses, IFN induction (adjusted $P$ value $=0.0028$ ) and type I IFN induction and signaling during SARS-CoV-2 infection (adjusted $P$ value $=0.0098$ ). In addition, among Gene Ontology and Reactome pathways, several gene sets were also significantly enriched. Notably, among enriched pathways were those related to IFN type I or antiviral response (Extended Data Fig. 2a).

ACE2. Angiotensin-converting enzyme 2 (ACE2) converts angiotensin II into angiotensin (1-7) as part of the renin-angiotensinaldosterone system and more notably, is the viral receptor for SARS-CoV-2. We identified seven cis-eQTLs in seven tissues (Supplementary Table 13) for ACE2, which are strongly correlated $\left(r^{2}>0.75\right.$ in 1000G EUR; Supplementary Table 14) with rs4830976 being the eQTL in the region most strongly associated with COVID-19 hospitalization.

pQTLs for ACE2. Stepwise conditional analysis for plasma ACE2 measured by Olink revealed one pQTL, rs5935998 $\left(P=1.45 \times 10^{-21}\right)$, which is in high LD with a previously reported cis-pQTL (rs12558179) for ACE2 $\left(r^{2}=0.89 \text { in } 1000 \mathrm{G} \text { EUR }\right)^{22}$ and a secondary suggestive signal ( $r s 4646156, P=3.20 \times 10^{-7}$ ). rs5935998 and rs4646156 are concordant in their effect on COVID-19 hospitalization (higher ACE2 levels corresponds to higher risk of COVID-19 hospitalization for both) resulting in a strong, positive MR association (MR $\beta$-coefficient: 0.34 ; $95 \%$ CI: $\left.0.17-0.51 ; P=8.1 \times 10^{-5}\right)$. Although neither rs5935998 or rs4646156 strongly colocalized with COVID-19 hospitalization (PP.H4 =0.49 for rs5935998, PP.H4 $=0.08$ for rs4646156, Extended Data Fig. 3), the two pQTLs, while statistically independent, are mildly correlated ( $r^{2}=0.20$ in $1000 \mathrm{G}$ EUR), which can make colocalization difficult to interpret ${ }^{23}$. One possible explanation is that these two pQTLs confer an effect on COVID-19 hospitalization that converges on the rs4830976-LD-block, as both are moderately correlated with $\mathrm{rs} 4830976\left(r^{2}=0.32\right.$ for $\mathrm{rs} 5935998$, $r^{2}=0.42$ for rs4646156 in 1000G EUR, Extended Data Fig. 3)

Phenome-wide scan of rs4830976. rs4830976 is associated $\left(P<3.96 \times 10^{-5}\right)$ with and colocalized (PP.H4 $\left.>0.80\right)$ with expression of nearby genes CA5B, CLTRN (also known as TMEM27) and VEGFD (Supplementary Table 15) in at least one tissue, indicating that this variant may be instrumental in gene expression beyond $A C E 2$. However, given the biological prior that ACE2 acts as the receptor of SARS-CoV-2, ACE2 is probably more likely than $C A 5 B$, CLTRN or VEGFD to be responsible for COVID-19 hospitalization. There were no other reported phenome-wide scan results at $P<3.96 \times 10^{-5}$ for rs4830976, which is at least in part due to the lack of reported X-chromosome results from a large proportion of GWAS.

Pathway enrichment analysis of rs4830976. Exploring the landscape of genes differentially expressed according to genotype in GTEx v.8, we observed 1,397 genes differentially expressed at a nominal $P$ value $<0.05$. Overrepresentation analysis identified 238 significantly enriched biological pathways among differentially expressed genes (Extended Data Fig. 2b). Among these, signaling by ILs, 


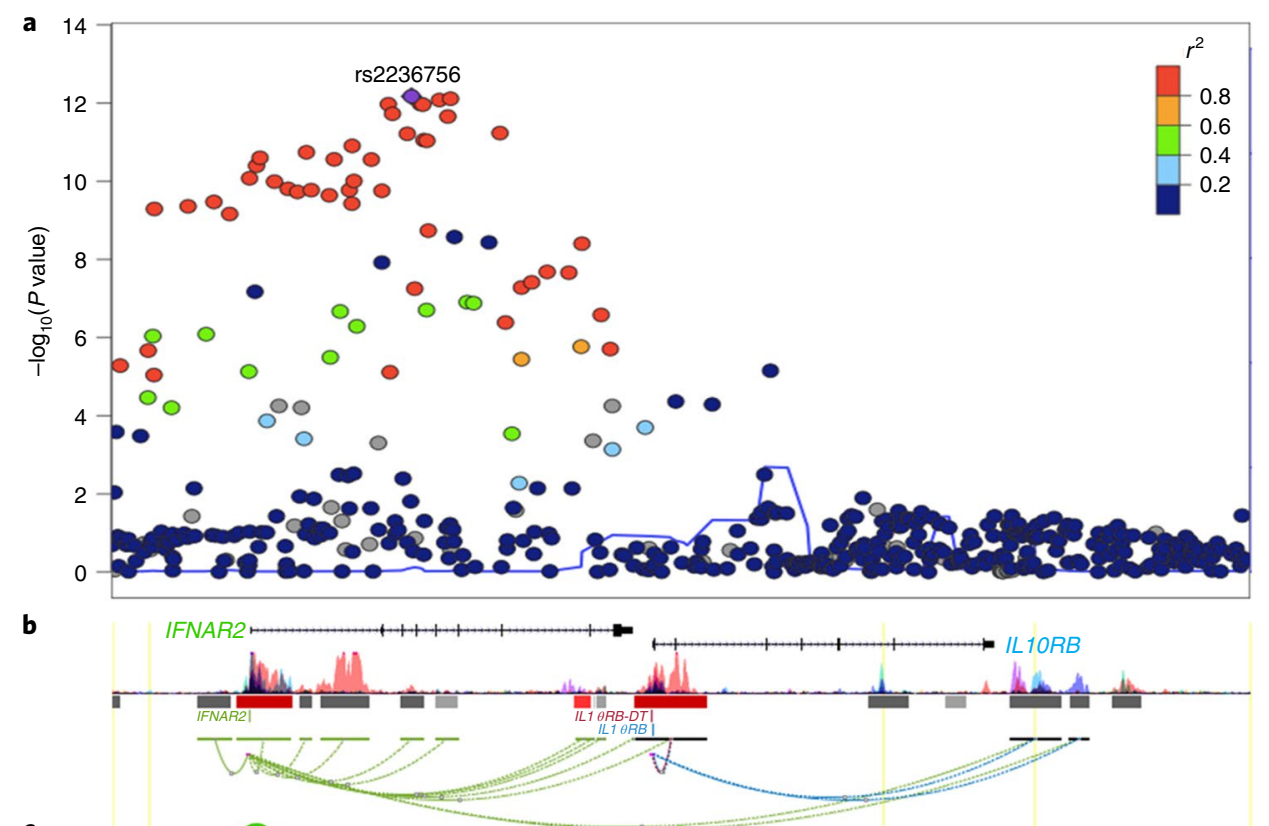

c

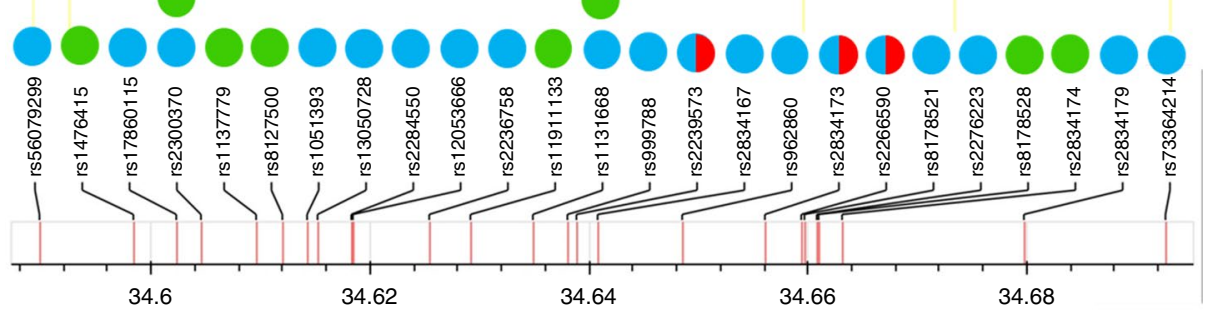

d

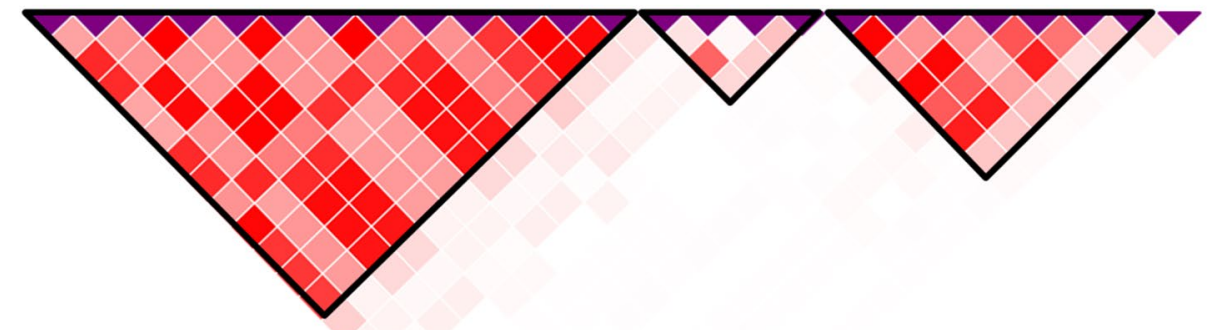

Fig. 3 | Genomic context, local association plot and LD structure of the IFNAR2/IL10RB region. $\mathbf{a}$, Local association plot $(N=1,377,758)$ of the interval defined by all unique eQTLs for IL1ORB or IFNAR2. Color code represents the degree of LD with the most associated marker in 1000G EUR. b, Genomic context of the region. Coding genes are represented by the refseq transcript. Bars represent epigenome roadmap-layered H3K27 acetylation markers. Connecting lines represent $\mathrm{Hi}-\mathrm{C}$ interactions. c, Set of rsIDs used as proposed instruments for MR analysis. Color code represents instruments for IL1ORB (blue circles), IFNAR2 (green circles). Red half-circles represent pQTLs for IL-1ORB. d, LD structure and blocks defined using European populations from 1000G EUR.

regulation of cytokine production and antigen processing and presentation might prove biologically relevant in COVID-19 infection.

\section{Discussion}

To identify drug-repurposing opportunities to inform trials against COVID-19, we conducted a large-scale MR analysis of protein and gene expression data. We first updated the 'actionable' genome to an enlarged set of 1,263 human proteins and provided evidence for 700 of these as targets for drugs with some potential relevance to COVID-19. By investigating more than 1,000 potential targets using several of the largest currently available human genetic datasets, we provide evidence for drug targets of type I IFNs (IFNAR2) and ACE2 modulators (ACE2) as priority candidates for evaluation in randomized trials of early management in COVID-19.
Our finding that ACE2 may play an important role in COVID-19 is unsurprising given its well-known relevance to SARS-CoV-2. As ACE2 acts as the primary receptor for SARS-CoV-2, increased expression of ACE2 has been hypothesized to lead to increased susceptibility to infection. ACE2 plays a vital role in the renin-angiotensin-aldosterone system signaling pathway, providing negative regulation through the conversion of angiotensin II to angiotensin 1-7. This action has anti-inflammatory and cardioprotective effects $^{24}$ and plays a protective role in acute respiratory distress syndrome ${ }^{25}$. ACE2 is a single-pass membrane protein but can be cleaved from the membrane to a soluble form which retains the enzymatic function to cleave angiotensin II. It has therefore been hypothesized that administration of human recombinant soluble ACE2 (hrsACE2) could be an effective treatment for COVID-19, 
a

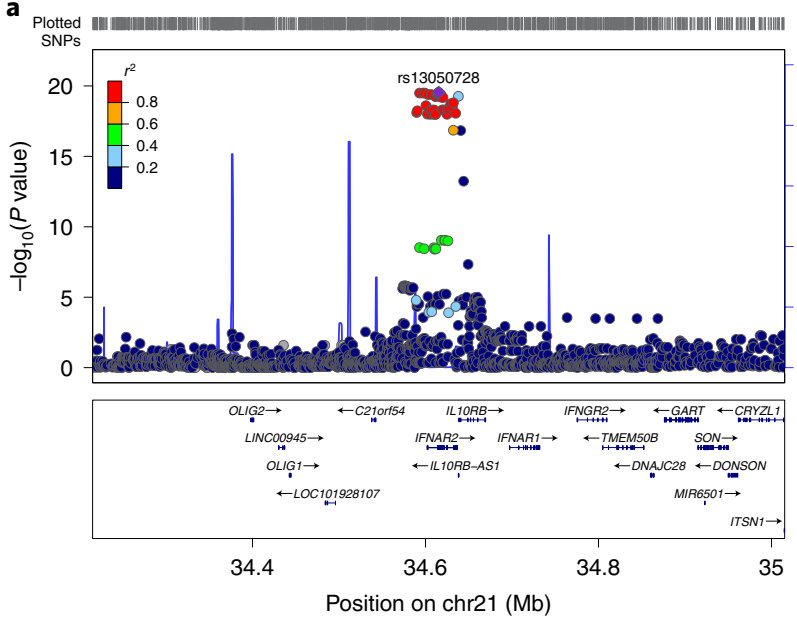

c

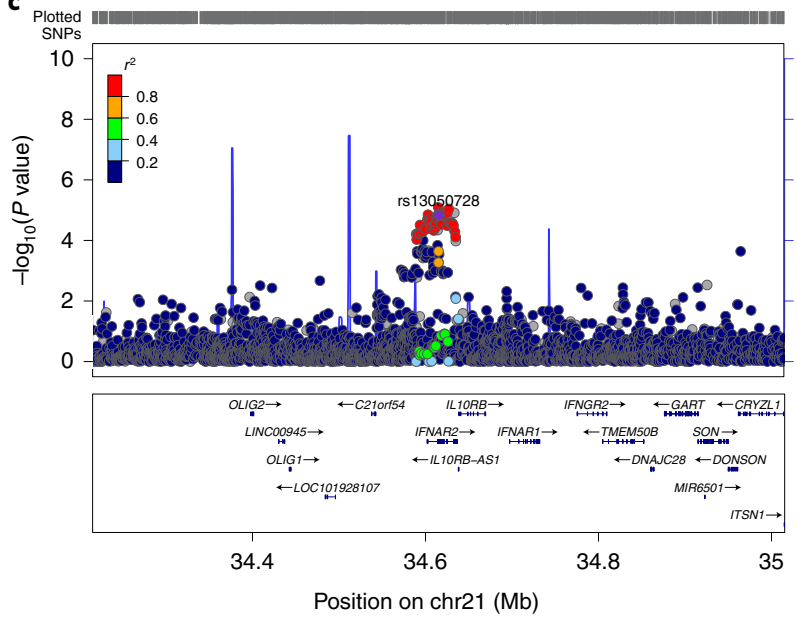

b

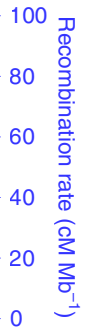

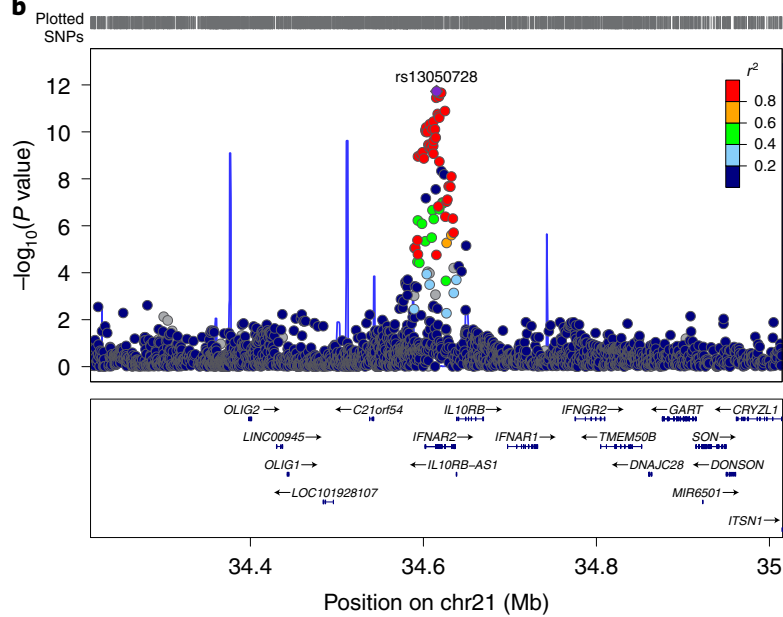

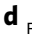

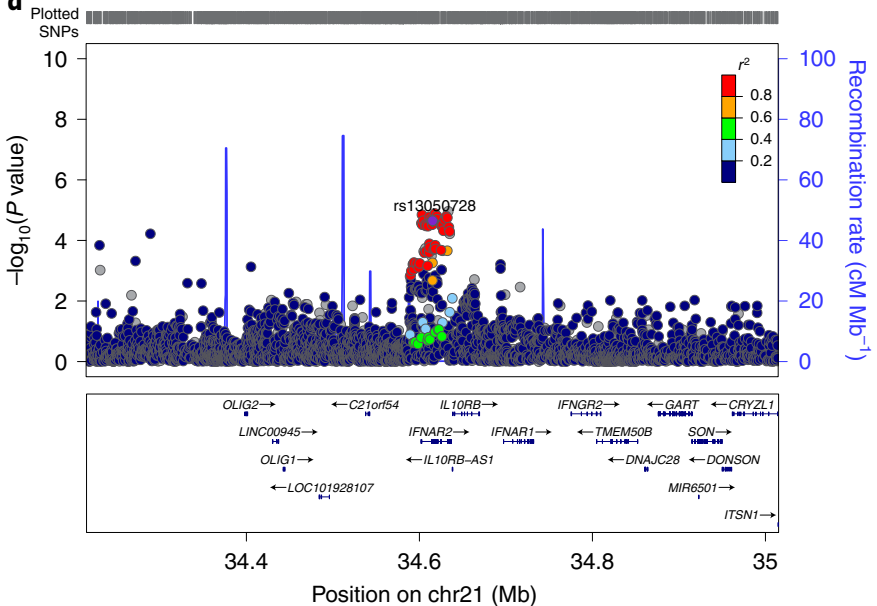

Fig. 4 | Regional association plots of the IFNAR2/IL1ORB locus. Regional association plots for $\mathbf{a}$, IL10RB gene expression in tibial nerve tissue from GTEx $(N=532)$, b, COVID-19 hospitalization from HGI and MVP transancestry meta-analysis $(N=1,377,758)$ c,d, VEGFR2 and TPSG1, respectively, measured by SomaLogic in 3,301 INTERVAL participants. All show the correlation (1000G EUR ancestry) for rs 13050728 , the cis-eQTL most associated with COVID-19 hospitalization in the IL1ORB-IFNAR2 region. All colocalize with each other (PP.H4 > 0.96 for all).

through distinct mechanisms in two phases of COVID-19. First, hrsACE2 can bind the viral spike glycoprotein of SARS-CoV-2, which could prevent cellular uptake of SARS-CoV-2 by reducing binding to the membrane-bound form of ACE2 (early phase). This suggestion is supported by the finding that APN01, a hrsACE2 therapeutic, showed a strong reduction in SARS-CoV-2 viral load ${ }^{26}$ and enhanced the benefit of remdesivir ${ }^{27}$ in primate kidney epithelial (Vero) cells and human kidney organoids. In the later phase, hrsACE2 could reduce sequelae of SARS-CoV-2 infection by reducing inflammation in the lungs and other infected tissues. A case report of a hospitalized COVID-19 patient supports this hypothesis by showing that 7-d administration of APN01 was associated with a reduction in SARS-CoV-2 viral load and inflammatory markers ${ }^{28}$. APN01 is currently being tested in a phase II trial to reduce mortality and invasive mechanical ventilation in 200 hospitalized patients with COVID-19 (https://ClinicalTrials.gov/show/NCT04335136). Notably, a recent report showed that expression of a truncated ACE2 isoform, dACE2, which poorly binds with SARS-CoV-2 spike protein, is stimulated by type I, II and III IFNs in human ileum organoids ${ }^{29}$.

One of the main challenges of our analysis was to determine whether IFNAR2 or IL10RB (or both) was driving the association with COVID-19 hospitalization, given that they share cis-eQTLs used as proposed instruments for our MR analysis. Multiple lines of evidence indicate that IFNAR2 appears to be primarily responsible for the signal observed. First, our phenome-wide scan using the lead IFNAR2/IL10RB cis-eQTL reproduced known effects of type I IFNs (the therapeutic target of IFNAR2) on VEGFR2 and TPSG1 (refs. ${ }^{18-20}$ ). Second, our pathway enrichment analysis using the same eQTL revealed pathways associated with type I IFN receptor (IFNAR2) signaling. Last, three independent cis-pQTLs that are also cis-eQTLs for IL10RB did not show evidence of association with COVID-19, suggesting that plasma IL-10RB concentrations are less likely to be etiologically relevant to COVID-19.

Evidence of a role for type I IFN in COVID-19 is rapidly emerging. Studies using in vitro (A549 pulmonary cell lines), animal (ferrets) and ex vivo (human lung tissue) models have all shown lower expression of genes encoding type I IFNs after exposure to SARS-CoV-2 compared to other respiratory viruses ${ }^{30,31}$. This has been confirmed in vivo by studies showing impaired type I IFN response, including almost no IFN- $\beta$ activity, in the peripheral blood of patients with severe COVID-19 compared to patients with mild-to-moderate COVID-19 (ref. ${ }^{32}$ ). More notably, lower levels of IFN- $\alpha-2$ among recently hospitalized patients with COVID-19 were associated with a substantial increase in the risk of progression to critical care, supporting our observation that lower genetically predicted IFNAR2 expression was associated with higher risk of COVID-19 hospitalization ${ }^{32}$. Additionally, auto-antibodies for 
type I IFNs were found in a much higher proportion of individuals with severe COVID-19 than those with asymptomatic or mild SARS-CoV-2 infection ${ }^{33}$.

Whole exome and genome sequencing studies on patients with severe COVID-19 have identified rare mutations that implicate type I IFN signaling. Zhang et al. ${ }^{34}$ found that patients with severe COVID-19 were enriched for rare variants predicted to cause loss of protein function at 13 genes involved in type I IFN response. A cases-series of four patients under the age of 35 years with severe COVID-19 found a rare loss-of-function mutation in TLR7 and decreased type I IFN signaling ${ }^{35}$. The GenOMICC study of imputed GWAS on severe COVID-19 identified signals that lie in the IFNAR2 gene $^{36}$.

Several in vitro studies have found a reduction in SARS-CoV-2 replication in multiple cell types (including animal and human) and human organoids after pretreatment with type I or III IFNs when compared with controls ${ }^{37-40}$ (Supplementary Table 16). Though these in vitro studies are encouraging, evidence from randomized trials for type I IFNs in early COVID-19 stages is limited. Hung et al. ${ }^{41}$ showed that randomization to a combination of IFN- $\beta-1 b$, ribavirin and lopinavir-ritonavir was superior to lopinavir-ritonavir alone in shortening the duration of viral shedding, alleviating symptoms and reducing the length of the hospital stay. Notably, these benefits were confined to a subgroup who were hospitalized within $7 \mathrm{~d}$ of onset of symptoms when IFN- $\beta-1 \mathrm{~b}$ was administered to the intervention arm. These results, together with our genetic findings on COVID-19 hospitalization and the established role of type I IFNs as first line of response against viral agents suggest recombinant type I IFN as potential intervention during early stages of COVID-19. To date, there is no large randomized trial on IFN- $\beta$ for early treatment of patients with COVID-19 who are at high risk of hospitalization.

Trial evidence on the use of IFN- $\beta$ in late stages of COVID-19 has emerged recently. The SOLIDARITY trial, which randomized 2,050 hospitalized patients with COVID-19 to IFN- $\beta-1 \mathrm{a}$, found no effect on mortality overall (relative risk $(\mathrm{RR})=1.16,95 \% \mathrm{CI}$ 0.96-1.39), but the trial was not powered to evaluate a possible trend across subgroups of COVID-19 severity at randomization $(\mathrm{RR}=1.40,95 \% \mathrm{CI} 0.82-2.40$ for those on ventilator, $\mathrm{RR}=1.13$, 95\% CI $0.86-1.50$ for those not ventilated but on oxygen and $\mathrm{RR}=0.80,95 \%$ CI $0.27-2.35$ in those with neither $)^{42}$. The Adaptive COVID-19 Treatment Trial 3 (ACTT-3) stopped enrollment of severely ill patients with COVID-19 for a trial on IFN- $\beta$ - $1 \mathrm{a}$ and remdesivir due to adverse events but continued enrolling patients with less severe disease ${ }^{43}$. The ACTT-2 found that baricitinib (an inhibitor of the JAK family of proteins, some of which are immediately downstream of IFNAR2) when administered to hospitalized patients with COVID-19 was beneficial in severe cases but not in moderate disease ${ }^{3}$. These findings indicate no role for the use of IFN- $\beta$ during late stages of COVID- 19 , when the cytokine storm is already established.

We are the first to implicate a causal role for ACE2 in COVID-19 manifestations using MR techniques; we have also implicated IFNAR2 in COVID-19, concordant with recent studies ${ }^{36,44}$. However, the current study notably complements and extends previous efforts by employing key approaches to protect against potential biases, strengthen causal inference and enhance understanding of potential mechanisms. First, in contrast to Liu et al. and the GenOMICC study, the current study involved several measures to minimize potential biases. We used colocalization methods to minimize the chances of false positive results due to confounding by LD. We reduced the possible impact of bias due to horizontal pleiotropy by restricting our proposed instruments to variants acting in cis and performing a phenome-wide scan to ensure instrumental variants were only associated/colocalized with gene expression of the tested gene or downstream phenotypes. When the possibility of horizontal pleiotropy was identified (for example IFNAR2 and IL10RB-sharing eQTLs), we addressed this using pQTL data and pathway enrichment analysis to disentangle mechanisms, ultimately showing that IFNAR2 is more likely to be the causal gene. Phenome-wide scans revealing effects on plasma proteins (VEGFR2 and TPSG1) that mimic known biology of type I IFN provides confidence that we are correctly instrumenting IFNAR2 and can identify on-target (harmful or beneficial) effects of administering type I IFN.

Second, our study had excellent statistical power, yielding strong MR associations for IFNAR2 $\left(P=9.8 \times 10^{-11}\right)$, increasing confidence in the validity of the much weaker signals for IFNAR2 reported in the GenOMICC study $(P=0.004)$, particularly as the earlier report had displayed evidence of confounding by LD ( $P$ for heterogeneity in dependent instruments (HIEDI) $=0.015)^{36}$. Indeed, compared to the analysis on COVID-19 hospitalization by Liu et al., our analysis contained more than double the number of cases ${ }^{44}$. Third, with our rigorous instrument selection process that used comprehensive datasets on gene expression and plasma protein levels, we were able to robustly evaluate over 1000 actionable drug targets, such as ACE2, which was not evaluated in the previous MR studies. Fourth, inclusion of MVP with HGI provided a more diverse population and identification of credible biological targets that were consistent across multiple ancestral groups.

Last, we provide an updated catalog of all actionable protein targets and drugs that are amenable to causal inference investigation through human genetics that can be applied to outcomes beyond COVID-19. For 700 proteins of the actionable genes, we also include information as to potential relevance to the treatment of COVID-19, which can help future studies to contextualize findings on COVID-19.

Our analysis also has limitations. Though we make use of instrumental variants from multiple data sources, they did not cover the entire actionable druggable genome or were derived from patients with COVID-19. Identifying the most relevant tissue or cell type can be challenging for interpreting MR analyses of gene expression. In our case, a relevant tissue could be one invaded by SARS-CoV-2, an organ associated with clinical complications of COVID-19, a tissue where the COVID-19-relevant protein is produced or a tissue that would be the likely site of action for the target drug. We opted to use a data-driven strategy that incorporates all tissues available in GTEx v.8. For IFNAR2, we recovered fibroblasts (the main cell type responsible for IFN- $\beta$ production), esophageal mucosa ${ }^{45}$ (a tissue invaded by SARS-CoV-2) and skeletal muscle ${ }^{46}$ (associated with the neurological manifestations of COVID-19). For ACE2, we recovered brain tissue, an organ known to be invaded by SARS-CoV-2 and associated with clinical manifestations ${ }^{47,48}$. Last, this work focused on cis-variants with an effect on gene expression and protein levels. We did not consider the full complexity of gene isoforms and splice single-nucleotide polymorphisms (SNPs), therefore missing mediation relationships that are isoform-specific. Also, we did not consider other pathways through which variants may affect disease, such as DNA methylation, histone modification, chromatin accessibility and others.

In conclusion, our transancestry $\mathrm{MR}$ analysis covering all actionable druggable genes identified two drug-repurposing opportunities (type I IFNs and hsrACE2) as interventions that need to be evaluated in adequately powered randomized trials to investigate their efficacy and safety for early management of COVID-19.

\section{Online content}

Any methods, additional references, Nature Research reporting summaries, source data, extended data, supplementary information, acknowledgements, peer review information; details of author contributions and competing interests; and statements of data and code availability are available at https://doi.org/10.1038/ s41591-021-01310-z. 
Received: 11 November 2020; Accepted: 5 March 2021;

Published online: 9 April 2021

\section{References}

1. Horby, P. et al. Dexamethasone in hospitalized patients with COVID-19preliminary report. N. Engl. J. Med. https://doi.org/10.1056/NEJMoa2021436 (2020).

2. Beigel, J. H. et al. Remdesivir for the treatment of COVID-19-final report. N. Engl. J. Med. https://doi.org/10.1056/NEJMoa2007764 (2020).

3. Kalil, A. C. et al. Baricitinib plus remdesivir for hospitalized adults with COVID-19. N. Engl. J. Med. https://doi.org/10.1056/NEJMoa2031994 (2020).

4. Nelson, M. R. et al. The support of human genetic evidence for approved drug indications. Nat. Genet. 47, 856-860 (2015).

5. Cohen, J. C., Boerwinkle, E., Mosley, T. H. Jr. \& Hobbs, H. H. Sequence variations in PCSK9, low LDL, and protection against coronary heart disease. N. Engl. J. Med. 354, 1264-1272 (2006).

6. Lopalco, L. CCR5: from natural resistance to a new anti-HIV strategy. Viruses 2, 574-600 (2010)

7. Finan, C. et al. The druggable genome and support for target identification and validation in drug development. Sci. Transl. Med. https://doi.org/10.1126/ scitranslmed.aag1166 (2017).

8. Swerdlow, D. I. et al. The interleukin-6 receptor as a target for prevention of coronary heart disease: a mendelian randomisation analysis. Lancet $\mathbf{3 7 9}$, 1214-1224 (2012).

9. The COVID-19 Host Genetics Initiative The COVID-19 Host Genetics Initiative, a global initiative to elucidate the role of host genetic factors in susceptibility and severity of the SARS-CoV-2 virus pandemic. Eur. J. Hum. Genet. 28, 715-718 (2020).

10. Gaziano, J. M. et al. Million veteran program: a mega-biobank to study genetic influences on health and disease. J. Clin. Epidemiol. 70, 214-223 (2016)

11. Labrecque, J. \& Swanson, S. A. Understanding the assumptions underlying instrumental variable analyses: a brief review of falsification strategies and related tools. Curr. Epidemiol. Rep. 5, 214-220 (2018).

12. Lonsdale, J. \& et al. The Genotype-Tissue Expression (GTEx) project. Nat. Genet. 45, 580-585 (2013).

13. Sun, B. B. et al. Genomic atlas of the human plasma proteome. Nature $\mathbf{5 5 8}$, 73-79 (2018)

14. Pietzner, M. et al. Genetic architecture of host proteins involved in SARS-CoV-2 infection. Nat. Commun. 11, 6397 (2020).

15. Borden, E. C. et al. Interferons at age 50: past, current and future impact on biomedicine. Nat. Rev. Drug Disco. 6, 975-990 (2007).

16. Emilsson, V. et al. Co-regulatory networks of human serum proteins link genetics to disease. Science 361, 769-773 (2018).

17. Staley, J. R. et al. PhenoScanner: a database of human genotype-phenotype associations. Bioinformatics 32, 3207-3209 (2016).

18. von Marschall, Z. et al. Effects of interferon- $\alpha$ on vascular endothelial growth factor gene transcription and tumor angiogenesis. J. Natl Cancer Inst. 95, 437-448 (2003).

19. Jia, H. et al. Endothelial cell functions impaired by interferon in vitro: insights into the molecular mechanism of thrombotic microangiopathy associated with interferon therapy. Thromb. Res. 163, 105-116 (2018).

20. Casassus, P. et al. Treatment of adult systemic mastocytosis with interferon- $\alpha$ : results of a multicentre phase II trial on 20 patients. Br. J. Haematol. 119, 1090-1097 (2002).

21. Swanson, S. A., Tiemeier, H., Ikram, M. A. \& Hernán, M. A. Nature as a trialist?: deconstructing the analogy between Mendelian randomization and randomized trials. Epidemiology 28, 653-659 (2017).

22. Nelson, C. P. et al. Genetic associations with plasma angiotensin converting enzyme 2 concentration: potential relevance to COVID-19 risk. Circulation 142, 1117-1119 (2020)

23. Wallace, C. Eliciting priors and relaxing the single causal variant assumption in colocalisation analyses. PLoS Genet. 16, e1008720 (2020).

24. Hemnes, A. R. et al. A potential therapeutic role for angiotensin-converting enzyme 2 in human pulmonary arterial hypertension. Eur. Respir. J. https:// doi.org/10.1183/13993003.02638-2017 (2018).
25. Kuba, K., Imai, Y., Rao, S., Jiang, C. \& Penninger, J. M. Lessons from SARS: control of acute lung failure by the SARS receptor ACE2. J. Mol. Med. 84 814-820 (2006).

26. Monteil, V. et al. Inhibition of SARS-CoV-2 infections in engineered human tissues using clinical-grade soluble human ACE2. Cell 181, 905-913 (2020).

27. Monteil, V. et al. Human soluble ACE2 improves the effect of remdesivir in SARS-CoV-2 infection. EMBO Mol. Med. 13, el3426 (2020).

28. Zoufaly, A. et al. Human recombinant soluble ACE2 in severe COVID-19. Lancet Respir. Med. https://doi.org/10.1016/S2213-2600(20)30418-5 (2020).

29. Onabajo, O. O. et al. Interferons and viruses induce a novel truncated ACE2 isoform and not the full-length SARS-CoV-2 receptor. Nat. Genet. 52, 1283-1293 (2020)

30. Blanco-Melo, D. et al. Imbalanced host response to SARS-CoV-2 drives development of COVID-19. Cell 181, 1036-1045 (2020).

31. Chu, H. et al. Comparative replication and immune activation profiles of SARS-CoV-2 and SARS-CoV in human lungs: an ex vivo study with implications for the pathogenesis of COVID-19. Clin. Infect. Dis. 71, 1400-1409 (2020).

32. Hadjadj, J. et al. Impaired type I interferon activity and inflammatory responses in severe COVID-19 patients. Science 369, 718-724 (2020)

33. Bastard, P. et al. Auto-antibodies against type I IFNs in patients with life-threatening COVID-19. Science https://doi.org/10.1126/science.abd4585 (2020).

34. Zhang, Q. et al. Inborn errors of type I IFN immunity in patients with life-threatening COVID-19. Science https://doi.org/10.1126/science.abd4570 (2020).

35. van der Made, C. I. et al. Presence of genetic variants among young men with severe COVID-19. JAMA 324, 1-11 (2020).

36. Pairo-Castineira, E. et al. Genetic mechanisms of critical illness in COVID-19. Nature https://doi.org/10.1038/s41586-020-03065-y (2020).

37. Lokugamage, K. G. et al. Type I interferon susceptibility distinguishes SARS-CoV-2 from SARS-CoV. J. Virol. 94, e01410-e01420 (2020).

38. Mantlo, E., Bukreyeva, N., Maruyama, J., Paessler, S. \& Huang, C. Antiviral activities of type I interferons to SARS-CoV-2 infection. Antivir. Res. 179, $104811(2020)$

39. Clementi, N. et al. Interferon- $\beta$-1a inhibition of severe acute respiratory syndrome-coronavirus 2 in vitro when administered after virus infection. $J$ Infect. Dis. 222, 722-725 (2020).

40. Dinnon, K. H. et al. A mouse-adapted model of SARS-CoV-2 to test COVID-19 countermeasures. Nature 586, 560-566 (2020).

41. Hung, I. F. et al. Triple combination of interferon- $\beta-1 b$, lopinavir-ritonavir, and ribavirin in the treatment of patients admitted to hospital with COVID-19: an open-label, randomised, phase 2 trial. Lancet 395, 1695-1704 (2020)

42. WHO SOLIDARITY Trial Consortium. Repurposed antiviral drugs for COVID-19: interim WHO solidarity trial results. N. Engl. J. Med. https://doi. org/10.1056/NEJMoa2023184 (2021).

43. FDA News. NIAID stops COVID-19 trial enrollment over adverse events. https://www.fdanews.com/articles/199319-niaid-stops-covid-19-trial-enrollm ent-over-adverse-events (2020)

44. Liu, D. et al. Mendelian randomization analysis identified genes pleiotropically associated with the risk and prognosis of COVID-19. J. Infect. https://doi.org/10.1016/j.jinf.2020.11.031 (2021).

45. Xiao, F. et al. Evidence for gastrointestinal infection of SARS-CoV-2. Gastroenterology 158, 1831-1833 (2020).

46. Mao, L. et al. Neurologic manifestations of hospitalized patients with coronavirus disease 2019 in Wuhan, China. JAMA Neurol. 77, 683-690 (2020).

47. Song, E. et al. Neuroinvasion of SARS-CoV-2 in human and mouse brain. $J$. Exp. Med. https://doi.org/10.1084/jem.20202135 (2021).

48. Puelles, V. G. et al. Multiorgan and renal tropism of SARS-CoV-2. N. Engl. J. Med. 383, 590-592 (2020).

Publisher's note Springer Nature remains neutral with regard to jurisdictional claims in published maps and institutional affiliations.

(๑) The Author(s), under exclusive licence to Springer Nature America, Inc. 202

'Massachusetts Veterans Epidemiology Research and Information Center (MAVERIC), VA Boston Healthcare System, Boston, MA, USA. ${ }^{2}$ BHF Cardiovascular Epidemiology Unit, Department of Public Health and Primary Care, University of Cambridge, Cambridge, UK. ${ }^{3}$ Central RNA Lab, Istituto Italiano di Tecnologia, Genoa, Italy. ${ }^{4}$ Department of Pathology and Laboratory Medicine, David Geffen School of Medicine, University of California Los Angeles, Los Angeles, CA, USA. '5aboratory of Genetics and Molecular Cardiology, Heart Institute, University of São Paulo, São Paulo, Brazil. ${ }^{6}$ Genetics Department, Harvard Medical School, Harvard University, Boston, MA, USA. ${ }^{7}$ Chemical Biology, European Molecular Biology Laboratory, European Bioinformatics Institute, Hinxton, UK. ${ }^{8}$ Department of Epidemiology, Erasmus Medical Center, Rotterdam, the Netherlands. 'Louis Stokes Cleveland VA Medical Center, Cleveland, OH, USA. ${ }^{10}$ Department of Population and Quantitative Health Sciences, Case Western Reserve University and Louis Stoke, Cleveland VA, Cleveland, OH, USA. "The Corporal Michael J. Crescenz VA Medical Center, Perelman School of Medicine at the University of Pennsylvania, Philadelphia, PA, USA. ${ }^{2}$ Department of Medicine, Perelman School of Medicine at the University of Pennsylvania, Philadelphia, PA, USA. 
${ }^{13}$ Biostatistics, School of Public Health, Boston University, Boston, MA, USA. ${ }^{14}$ European Molecular Biology Laboratory, European Bioinformatics Institute, Hinxton, UK. ${ }^{15}$ Department of Medical Sciences, Uppsala University, Uppsala, Sweden. ${ }^{16}$ Berlin Institute of Health, Charité University Medicine Berlin, Berlin, Germany. ${ }^{17} \mathrm{MRC}$ Epidemiology Unit, University of Cambridge, Cambridge, UK. ${ }^{18}$ Institute of Health Informatics, University College London, London, UK. ${ }^{19}$ Health Data Research, University College London, London, UK. ${ }^{20}$ British Heart Foundation Centre of Research Excellence, University of Cambridge, Cambridge, UK. ${ }^{21} \mathrm{Health}$ Data Research UK Cambridge, Wellcome Genome Campus and University of Cambridge, Cambridge, UK. ${ }^{22}$ Department of Public Health and Primary Care, University of Cambridge, Cambridge, UK. ${ }^{23} \mathrm{MRC}$ Biostatistics Unit, University of Cambridge, Cambridge, UK. ${ }^{24} \mathrm{Centre}$ for Inflammatory Disease, Dept of Immunology and Inflammation, Imperial College, London, UK. ${ }^{25}$ National Institute for Health Research Blood and Transplant Research Unit in Donor Health and Genomics, University of Cambridge, Cambridge, UK. ${ }^{26}$ National Institute for Health Research Cambridge Biomedical Research Centre, University of Cambridge and Cambridge University Hospitals, Cambridge, UK. ${ }^{27}$ VA Informatics and Computing Infrastructure, VA Salt Lake City Health Care System, Salt Lake City, UT, USA. ${ }^{28}$ Department of Internal Medicine, Epidemiology, University of Utah, Salt Lake City, UT, USA. ${ }^{29}$ Department of Epidemiology and Biostatistics, University of Arizona, Tucson, AZ, USA. ${ }^{30}$ Phoenix VA Health Care System, Phoenix, AZ, USA. ${ }^{31}$ Center for Population Genomics, Massachusetts Veterans Epidemiology Research and Information Center (MAVERIC), VA Boston Healthcare System, Boston, MA, USA. ${ }^{32}$ Cardiology, VA Boston Healthcare System, Boston, MA, USA. ${ }^{33}$ Medicine, Brigham and Women's Hospital, Harvard Medical School, Boston, MA, USA. ${ }^{34}$ Epidemiology Research and Information Center (ERIC), VA Palo Alto Health Care System, Palo Alto, CA, USA. ${ }^{35}$ Department of Medicine, Stanford University School of Medicine, Palo Alto, CA, USA. ${ }^{36}$ MIRECC, Durham VA Medical Center, Durham, NC, USA. ${ }^{37}$ Department of Psychiatry and Behavioral Sciences, Duke University School of Medicine, Durham, NC, USA. ${ }^{38}$ Office of Research and Development, Department of Veterans Affairs, Washington, DC, USA. ${ }^{39}$ VA Tennessee Valley Healthcare System, Nashville, TN, USA. ${ }^{0}$ Nephrology \& Hypertension, Vanderbilt University, Nashville, TN, USA. ${ }^{41}$ The Corporal Michael J. Crescenz VA Medical Center, Philadelphia, PA, USA. ${ }^{42}$ Atlanta VA Health Care System, Decatur, GA, USA. ${ }^{43}$ Department of Epidemiology, Emory University Rollins School of Public Health, Atlanta, GA, USA. ${ }^{44}$ Medicine, Cardiovascular, VA Boston Healthcare System and Brigham \& Women's Hospital, Boston, MA, USA. ${ }^{45}$ Department of Veterans Affairs, Tennessee Valley Healthcare System, Vanderbilt University, Nashville, TN, USA. ${ }^{46}$ Medicine, Epidemiology, Vanderbilt Genetics Institute, Vanderbilt University Medical Center, Nashville, TN, USA. ${ }^{47}$ Division of Aging, Brigham and Women's Hospital, Harvard Medical School, Boston, MA, USA. *A list of authors and their affiliations appears at the end of the paper.

凶e-mail: asb38@medschl.cam.ac.uk; jpcasasromero@bwh.harvard.edu

\section{VA Million Veteran Program COVID-19 Science Initiative}

Yuk-Lam Ho', Sudha K. Iyengar, ${ }^{90}$, Nicole M. Kosik', James E. Peters ${ }^{21,24}$, Kristine E. Lynch ${ }^{27,28,}$ Scott L. DuVall ${ }^{27,28}$, Helene Garcon', Lauren O. Thomann', Jennifer E. Huffman ${ }^{31}$, Christopher J. O'Donnell ${ }^{32,33}$, Philip S. Tsao ${ }^{34,35}$, Jean C. Beckham ${ }^{36,37}$, Saiju Pyarajan', Sumitra Muralidhar ${ }^{38}$, Grant D. Huang ${ }^{38}$, Rachel Ramoni ${ }^{38}$, Adriana M. Hung ${ }^{39,40}$, Kyong-Mi Chang ${ }^{12,41}$, Yan V. Sun ${ }^{42,43}$, Todd L. Edwards ${ }^{45,46}$, Kelly Cho ${ }^{1,47}$, J. Michael Gaziano ${ }^{1,47}$ and Juan P. Casas ${ }^{1,47}$ 


\section{Methods}

Identification of actionable druggable genes suitable for repurposing against COVID-19. Information about drugs and clinical candidates and their therapeutic targets, was obtained from the ChEMBL database (release 26 (ref. ${ }^{49}$ ) Supplementary Methods). For the purposes of our COVID-19 drug-repurposing efforts, actionable proteins were defined as those that are therapeutic targets of approved drugs and clinical candidates or are potential targets of approved drugs. Therapeutic targets were identified from the drug mechanism of action information in ChEMBL and linked to their component proteins. Each protein was assigned a confidence level based on the type and size of target annotated and the resulting list was filtered to remove nonhuman proteins and those with lower confidence assignments (cases where the therapeutic target consists of more than ten proteins or the protein is known to be a nondrug-binding subunit of a protein complex). For approved drugs, additional potential human target proteins were identified from pharmacological assay data in ChEMBL with recorded affinity/ efficacy measurements $\leq 100 \mathrm{nM}$ (represented by a pChEMBL value $\geq 7$ ).

A total of 1,263 unique human proteins were identified as 'actionable' from data available in ChEMBL. These consisted of 531 proteins that are therapeutic targets of approved drugs, 381 additional proteins that are therapeutic targets of clinical candidates and 351 additional proteins that are bound by approved drugs, but not annotated as the therapeutic targets. While the biological relevance of the latter group of targets in the context of the approved drug indications may be unclear, the high affinity/efficacy measurements suggest the drug should be capable of modulating these proteins, should they be found to be relevant to COVID-19 (although likely not in a selective manner). Proteins were further annotated with biological and drug information relating to their potential role in SARS-CoV-2 infection (Supplementary Methods) such as change in abundance during infection, interaction with viral proteins or the activity of drugs in antiviral cell-based assays. Of the 1,263 actionable proteins identified previously, 300 were annotated as biologically relevant in SARS-CoV-2 infection and 547 were targets of drugs with some evidence of COVID-19 relevance from cell-based assays, clinical trials or the ATC classification (Supplementary Table 2)

Selection of proposed instruments. eQTL proposed instruments. We proposed eQTL instruments using raw data from GTEx v.8 by performing conditional analysis on normalized gene expression in European ancestry individuals in 49 tissues that had at least 70 samples. eQTLs were derived in all 49 tissues (that is we did not restrict it to tissues we thought most relevant to COVID-19) because the biological relevance of tissues to SARS-CoV-2 infection is still rapidly evolving. We used Matrix eQTL ${ }^{50}$ and followed the same procedure as outlined by the GTEx consortium (https:// gtexportal.org/home/). Briefly, after filtering the genotypes (genotype missingness $<0.05$, minor allele frequency $<0.01$, Hardy-Weinberg equilibrium $<0.000001$, removing ambiguous SNPs), within each tissue, we performed GWAS between variants and gene expression adjusting for sex, the first five principal components of European genetic ancestry, PEER factors, sequencing platform and protocol. To identify independent eQTLs, we performed conditional analysis in regions around associations that fell below genome-wide (GW) significance, additionally adjusting for the peak variant if there exists an association reaching a $P$ value of $5.00 \times 10^{-8}$. Cis-eQTLs were defined as GW-significant $\left(P<5.00 \times 10^{-8}\right)$ associations within $1 \mathrm{Mb}$ on either side of the encoded gene. To convert from build 38 to build 37, we used the table available from the GTEx consortium for all variants genotyped in GTEx v.8 and hg19 liftover, (https://storage.googleapis.com/gtex analysis_v8/reference/ GTEx_Analysis_2017-06-05_v8_WholeGenomeSeq_838Indiv_Analysis_Freeze. lookup_table.txt.gz). In each tissue, multiple GW-significant $\left(P<5.00 \times 10^{-8}\right)$ eQTLs for the same gene were combined into a single instrument using inverse-variance weighting and fixed-effects meta-analysis across variant-level MR estimates for each variant, a standard two-sample MR approach. For example, for IL10RB expression in skeletal muscle tissue, there were two conditionally independent eQTLs (rs2300370 and rs2834167; Table 1); a variant-level MR-estimate was obtained for each by dividing the $\beta$-coefficient for COVID-19 hospitalization by the $\beta$-coefficient of the eQTL and dividing the standard error of the COVID-19 hospitalization estimate by the $\beta$-coefficient of the eQTL. The two variant-level MR estimates were then meta-analyzed using inverse-variance weighting and fixed effects to yield the final MR result. Instruments for expression of the same gene derived in different tissues were tested separately.

pQTL proposed instruments. We proposed pQTL instruments from two sources of publicly available data that reported conditionally independent pQTLs for proteins measured by the SomaLogic SomaScan ${ }^{51,52}$ platform: (1) Sun et al. ${ }^{13}$, who reported results for 2,994 proteins in 3,301 INTERVAL participants and (2) Pietzner et al. ${ }^{14}$, who reported results for 179 proteins in 10,708 participants of the Fenland cohort. In both, we restricted proposed instrumental variants to cis-pQTLs for actionable proteins, used a $P$ value threshold of $5 \times 10^{-8}$ and removed variants with minor allele frequency $<0.01$. MR was run independently for each data source (proposed instruments for the same protein in different platforms were tested against COVID-19 hospitalization independently).

Estimates for COVID-19 hospitalization. To generate outcome summary statistics, we meta-analyzed results from the MVP, an ongoing, prospective cohort recruiting from 63 Veterans Health Administration (VA) medical facilities (Supplementary Methods) and the Host Genetics Initiative ${ }^{9}$, a global collaboration to accumulate GWAS on COVID-19 infection and clinical manifestations.

In MVP, 1,062 COVID-19 cases (Supplementary Table 1) were identified between 1 March and 17 September 2020 using an algorithm developed by the VA COVID National Surveillance Tool. The National Surveillance Tool classified COVID-19 cases as positive or negative based on real-time PCR with reverse transcription (rRT-PCR) laboratory test results conducted at VA clinics, supplemented with natural language processing on clinical documents. The algorithm to identify patients with COVID-19 is continually updated to ensure new annotations of COVID-19 are captured from clinical notes, with chart reviews performed periodically to validate the algorithm ${ }^{53}$. COVID-19-related hospitalizations were defined as admissions from $7 \mathrm{~d}$ before up to $30 \mathrm{~d}$ after a patient's first positive test for SARS-CoV-2. We tested association between all our proposed genetic instruments and COVID-19 hospitalization (versus population controls) in MVP, adjusting for age, sex and the first ten principal components (PCs) in three mutually exclusive, ancestry-specific strata separately (European, African and Hispanic ancestry) using PLINK v.2 (analysis completed on 10 October 2020). We have previously provided a detailed description of the genotype data quality control process ${ }^{54}$. The MVP received ethical and study protocol approval by the Veterans Affairs Central Institutional Review Board and informed consent was obtained for all participants.

We downloaded publicly available summary statistics for the B2 outcome from Host Genetic Initiative on 4 October 2020 (release 4 v.1). In total, HGI accumulated 6,492 cases of COVID-19 hospitalization through collaboration from 16 contributing studies (Supplementary Table 1), which were asked to define cases as 'hospitalized laboratory confirmed SARS-CoV-2 infection (RNA and/or serology based), hospitalization due to corona-related symptoms' versus population controls (https://docs.google.com/document/ d/1okamrqYmJfa35ClLvCt_vEe4PkvrTwggHq7T3jbeyCI/view) and use a model that adjusts for age, age ${ }^{2}$, sex, age $\times$ sex, PCs and study specific covariates (https://docs.google.com/document/d/16ethjgi4MzlQeO0KAW yDYyUHdB9kKbtfuGW4XYVKQg/view). Summary statistics ( $\beta$ s and standard errors) from the four analyses, MVP-European, MVP-African, MVP-Hispanic and COVID-19 HGI (HGI summary statistics were already meta-analyzed from GWAS that contributed to the HGI consortium) were meta-analyzed using METAL software ${ }^{55}$ with inverse-variance weighting and fixed effects.

Quantile-quantile plots of $P$ values from genome-wide association testing in MVP did not display any inflation of results in any ancestry-specific stratum (Supplementary Fig. 1). Additionally, $P_{\text {het }}$ values from the meta-analysis (output from METAL's 'analyze heterogeneity' command) were not inflated (Supplementary Fig. 2), indicating that there is little overall heterogeneity between estimates across ancestries within MVP and between MVP and HGI

Mendelian randomization and colocalization. We conducted MR analyses using the R package TwoSampleMR (https://mrcieu.github.io/TwoSampleMR/). We used fixed-effects, inverse-variance-weighted MR for proposed instruments that contain more than one variant and Wald ratio for proposed instruments with one variant. For proposed instruments with multiple variants, we also tested the heterogeneity across variant-level MR estimates, using the Cochrane Q method (mr_heterogeneity option in TwoSampleMR package). We defined significant MR results using a $P$ value threshold of $P<3.96 \times 10^{-5}(0.05$ Bonferroni-corrected for 1,263 actionable druggable genes) and identified a list of 'suggestive' actionable druggable targets that passed a threshold of $P<5.00 \times 10^{-4}$. For statistically significant MR results, we also performed colocalization ${ }^{56}$ between each eQTL and the transancestry meta-analysis on COVID-19 hospitalization using the moloc $\mathrm{R}$ package (https://github.com/clagiamba/moloc) with default priors (probability of shared causal variant for trait 1 and trait 2 is $P 1=P 2=1 \times 10^{-4}$, probability of shared causal variant across two traits is $P 12=1 \times 10^{-5}$ ). For example, if a proposed instrument contained two variants, we performed colocalization for the primary eQTL GWAS with COVID-19 hospitalization, as well as the secondary eQTL GWAS (eQTL GWAS after adjusting for peak variant from primary GWAS) with COVID-19 hospitalization. Statistically significant MR hits with posterior probability for hypothesis 4 (PP.H4) $>0.8$ (the probability of a shared causal variant) for a least one instrumental variant were then investigated further using the following analyses.

Identifying pQTLs using Olink assay. We performed stepwise conditional analysis to identify cis-pQTL proposed instruments for proteins that passed our significance and colocalization thresholds and were one of 354 unique proteins measured on four Olink ${ }^{57}$ panels (CVD1, CVD2, Inflammation and Neuro; Olink Target 96 \& Target 48 panels for protein biomarker discovery from https://www. olink.com/products/target/) in 4,998 INTERVAL participants ${ }^{13}$. INTERVAL is a prospective cohort study of $\sim 50,000$ blood donors recruited from 25 National Health Service Blood and Transplant centers in England. Participants were genotyped using the UK Biobank Affymetrix Axiom array, followed by phasing using SHAPEIT3 and imputation on the Sanger Imputation Server using a 1000G Phase 3-UK10K imputation panel. Alleles were tested against Olink proteins using SNPTEST v.2.5.2 and adjusted for age, sex, plate, time from blood draw to 
processing, season and the first five PCs. Conditional analysis was performed by adjusting for peak variants until no association fell below $5.00 \times 10^{-6}$.

Phenome-wide scan. We conducted a phenome-wide scan for variants with the following goals. First, we wanted to evaluate whether our proposed instruments could reproduce known phenotype associations (for example disease, biomarkers) ascribed to the drug that were due to on-target effects. Second, we wanted to identify whether our proposed instruments were associated with comorbidities associated with greater likelihood of SARS-CoV-2 testing or predictors of hospitalization in patients with COVID-19, as this could potentially highlight the presence of certain biases ${ }^{21}$. Also, for genes that were the target of licensed drugs, we checked whether the disease indication was also a risk factor for COVID-19 outcomes, as this might introduce a bias analogous to confounding by indication in $\mathrm{MR}$.

To accomplish these goals, we investigated proposed instruments for associations of a phenome-wide range of outcomes. We searched the GTEx ${ }^{12}$ portal (https://gtexportal.org/home/) for gene expression and Phenoscanner ${ }^{17}$ (http:// www.phenoscanner.medschl.cam.ac.uk/) for proteins, traits and diseases. We additionally queried variants in GWAS for 354 Olink proteins (described earlier) and all the proteins measured by the SomaScan platform (described by Sun et al. ${ }^{13}$ ) in 3,301 INTERVAL participants.

Characterizing downstream transcriptional consequences of associated loci. To confirm specificity of identified loci and to better explore their most important downstream transcriptional consequences, we studied the transcriptional landscape modulation associated with the selected variants using GTEx v.8 data with representation of 49 different tissues. For this we have used rs 13050728 as the proxy of the IFNAR2/IL10RB locus and rs4830976 as the proxy of the ACE2 locus and conducted a differential gene-expression analysis for all transcripts available in GTEx v.8. After fitting models for all genes, enrichment pathway analysis was conducted to retrieve the most enriched pathways using both the differentially expressed gene list (through an overrepresentation analysis) and a gene set enrichment analysis framework (using the R package clusterProfiler ${ }^{58}$ ). For enrichment analyses we used the corpus from WikiPathways, Gene Ontology and Reactome.

Reporting Summary. Further information on research design is available in the Nature Research Reporting Summary linked to this article.

\section{Data availability}

GTEx project v.8 data are available at https://gtexportal.org/home/. CheMBL database data are available at https://www.ebi.ac.uk/chembl/. Fenland-SomaLogic protein GWAS data are available at https://omicscience.org/apps/covidpgwas/. HGI COVID-19 hospitalization summary statistics are available at https://www. covid19hg.org/. PhenoScanner results are available at http://www.phenoscanner. medschl.cam.ac.uk/.

\section{References}

49. Mendez, D. et al. ChEMBL: towards direct deposition of bioassay data. Nucleic Acids Res. 47, D930-D940 (2019).

50. Shabalin, A. A. Matrix eQTL: ultra fast eQTL analysis via large matrix operations. Bioinformatics 28, 1353-1358 (2012).

51. Rohloff, J. C. et al. Nucleic acid ligands with protein-like side chains: modified aptamers and their use as diagnostic and therapeutic agents. Mol. Ther. Nucleic Acids 3, e201 (2014).

52. Gold, L. et al. Aptamer-based multiplexed proteomic technology for biomarker discovery. PLoS ONE 5, e15004 (2010).

53. Chapman, A. B. et al. A Natural Language Processing System for National COVID-19 Surveillance in the US Department of Veterans Affairs. https:// www.aclweb.org/anthology/2020.nlpcovid19-acl.10 (Association for Computational Linguistics, 2020).

54. Hunter-Zinck, H. et al. Genotyping array design and data quality control in the million veteran program. Am. J. Hum. Genet 106, 535-548 (2020).

55. Willer, C. J., Li, Y. \& Abecasis, G. R. METAL: fast and efficient meta-analysis of genomewide association scans. Bioinformatics 26, 2190-2191 (2010).

56. Giambartolomei, C. et al. A Bayesian framework for multiple trait colocalization from summary association statistics. Bioinformatics 34, 2538-2545 (2018).

57. Lundberg, M., Eriksson, A., Tran, B., Assarsson, E. \& Fredriksson, S. Homogeneous antibody-based proximity extension assays provide sensitive and specific detection of low-abundant proteins in human blood. Nucleic Acids Res. 39, e102 (2011)

58. Yu, G., Wang, L. G., Han, Y. \& He, Q. Y. clusterProfiler: an R package for comparing biological themes among gene clusters. Omics 16, 284-287 (2012)
59. Wareham, N. Fenland Study (Version 1) Dataset. https://doi. org/10.22025/2017.10.101.00001 (MRC Epidemiology Unit, University of Cambridge, 2016)

\section{Acknowledgements}

We are grateful to the Host Genetic Initiative for making their data publicly available (full acknowledgements can be found at https://www.covid19hg.org/acknowledgements/). This research is based on data from the MVP, Office of Research and Development, VA and was supported by award no. MVP035. This research was also supported by additional Department of Veterans Affairs awards grant no. MVP001. This publication does not represent the views of the Department of Veteran Affairs or the US Government. Full acknowledgements for the VA MVP COVID-19 Science Initiative can be found in Supplementary Methods. C.G. has received funding from the European Union's Horizon 2020 Research and Innovation Program under the Marie Skłodowska-Curie grant agreement no. 754490, MINDED project. A.G., P.B. and A.R.L. are funded by the Member States of the European Molecular Biology Laboratory. I.B.-H. received funding from Open Targets (grant agreement OTAR-044). The Fenland study ${ }^{59}$ is funded by the Medical Research Council (MC_UU_12015/1); we are grateful to all the volunteers and to the General Practitioners and practice staff for assistance with recruitment; we thank the Fenland Study Investigators, Fenland Study Co-ordination team and the Epidemiology Field, Data and Laboratory teams; we further acknowledge support for genomics from the Medical Research Council (MC PC 13046); proteomic measurements were supported and governed by a collaboration agreement between the University of Cambridge and Somalogic. J.E.P. is supported by UK Research and Innovation Fellowship at Health Data Research UK (MR/S004068/2). L.R., N.H. and C.L. are supported by the Swedish Research Council. E.A. was supported by the EU/EFPIA Innovative Medicines Initiative Joint Undertaking BigData@Heart grant no. 116074 and by the British Heart Foundation Programme grant RG/18/13/33946. We thank A. Wood for feedback on statistical analyses used in the paper. We thank the INTERVAL Study investigators, co-ordination team and the epidemiology field, data and laboratory teams, who were supported by core funding from the UK Medical Research Council (MR/L003120/1), the British Heart Foundation (RG/13/13/30194; RG/18/13/33946), the National Institute for Health Research (NIHR) Cambridge Biomedical Research Centre (BRC-1215-20014) (the views expressed are those of the authors and not necessarily those of the NIHR or the Department of Health and Social Care) and the NIHR Blood and Transplant Research Unit in Donor Health and Genomics (NIHR BTRU-201410024). This work was also supported by Health Data Research UK, which is funded by the UK Medical Research Council, the Engineering and Physical Sciences Research Council, the Economic and Social Research Council, the Department of Health and Social Care (England), the Chief Scientist Office of the Scottish Government Health and Social Care Directorates, the Health and Social Care Research and Development Division (Welsh Government), the Public Health Agency (Northern Ireland), the British Heart Foundation and Wellcome. J.D. holds a British Heart Foundation Professorship and an NIHR Senior Investigator Award.

\section{Author contributions}

J.P.C., A.S.B. and J.M.G. conceived the study design. A.G., A.P.B. and A.R.L. defined the actionable genome and identified and curated drug information relating to SARS-CoV-2; P.B. and I.B.-H. provided biological annotation relating to SARS-CoV-2; C.G. performed stepwise conditional analysis on GTEx raw data; D.P. tested associations for COVID-19 in MVP; L.G. and J.H.Z. performed meta-analysis of HGI and MVP; L.G. performed MR analysis; L.G. and C.G. performed colocalization analyses; L.G. and B.P.P. performed conditional analysis on Olink proteins; L.G. and E.A. performed phenome-wide scans; A.C.P. performed pathway enrichment analysis; J.N.D., A.S.B. and J.E.P. provided INTERVAL data; several authors were involved in the curation of MVP data; L.G., C.G., A.C.P., A.G., D.P., A.S.B. and J.P.C. wrote the manuscript. J.P.C. oversaw all analyses. All authors critically reviewed the manuscript.

\section{Competing interests}

The authors declare no competing interests.

\section{Additional information}

Extended data is available for this paper at https://doi.org/10.1038/s41591-021-01310-Z.

Supplementary information The online version contains supplementary material available at https://doi.org/10.1038/s41591-021-01310-z.

Correspondence and requests for materials should be addressed to A.S.B. or J.P.C.

Peer review information Nature Medicine thanks David Evans, Steven Wolinsky and the other, anonymous, reviewer(s) for their contribution to the peer review of this work. Joao Monteiro was the primary editor on this article and managed its editorial process and peer review in collaboration with the rest of the editorial team.

Reprints and permissions information is available at www.nature.com/reprints. 


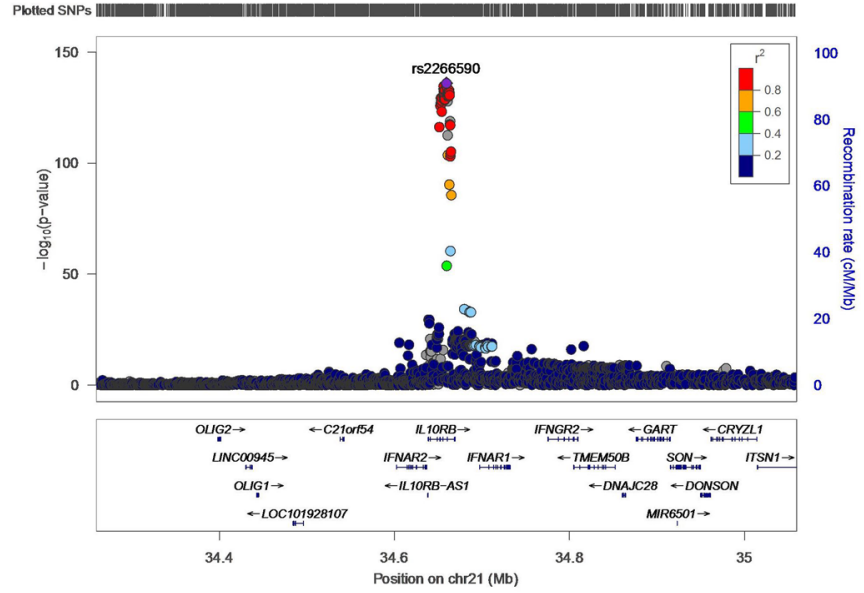

C

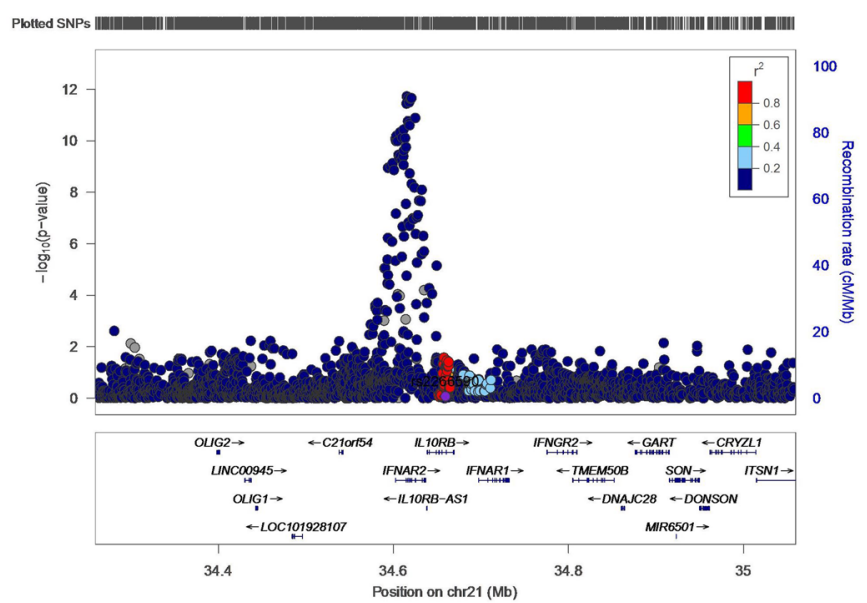

e

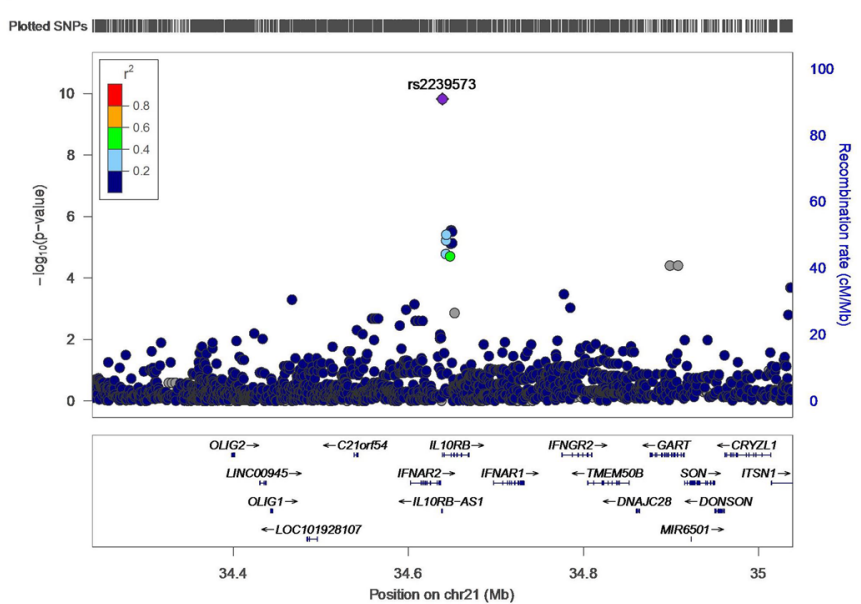

b

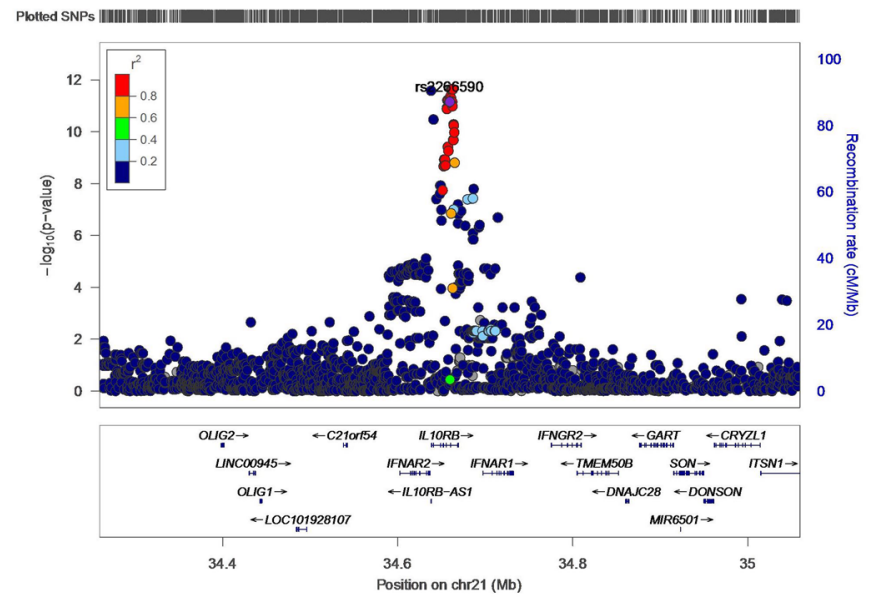

d

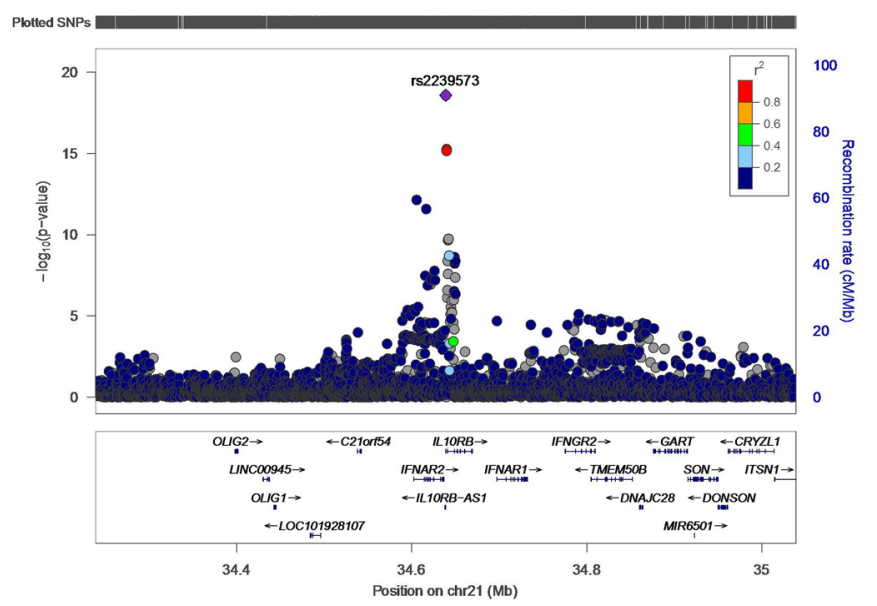

$\mathbf{f}$

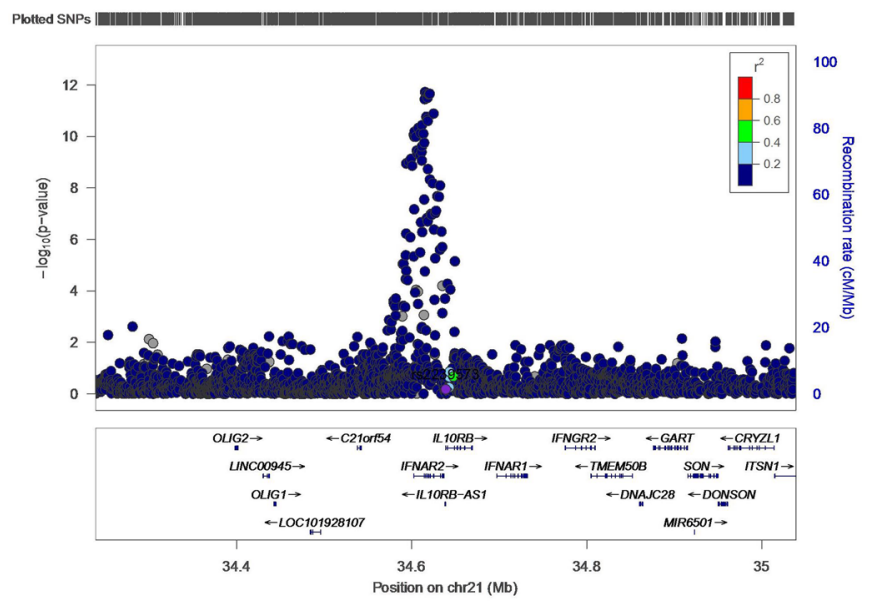

Extended Data Fig. 1 | Regional association plots for rs2266590 and rs2239573 for plasma IL-10RB, IL10RB gene expression, and COVID-19 hospitalization. This region was investigated further using IL-10RB pQTL data because it was available on an independent proteomic platform (Olink) and results using eQTL instruments for IL10RB passed our Mendelian randomization $P$ value threshold (0.05 Bonferroni-corrected for 1,263 actionable druggable genes) and colocalization threshold (PP.H4 > 0.8). a, rs2266590 as PQTL for plasma IL-10RB measured by Olink in 4,998 INTERVAL participants. b, rs2266590 as an eQTL for IL10RB expression tibial artery tissue (N=584). c, rs2266590 in COVID-19 hospitalization. d, rs2239573 as pQTL for plasma IL-10RB (after adjusting for rs2266590) measured by Olink in 4,998 INTERVAL participants. e, rs2239573 as an eQTL for IL10RB expression in whole blood ( $N=670)$. f, rs2239573 in COVID-19 hospitalization. a colocalizes with $\mathbf{b}$ (PP.H4=0.97), and $\mathbf{d}$ colocalizes with e (PP. $H 4=1.00$ ). The two variants highlighted in this figure ( $r s 2266590$ and rs2239573), which are associated with gene expression and plasma protein levels of IL-1ORB, are not associated with COVID-19 hospitalization ( $P=0.85$ for rs2266590, $P=0.66$ for rs2239573). 
a

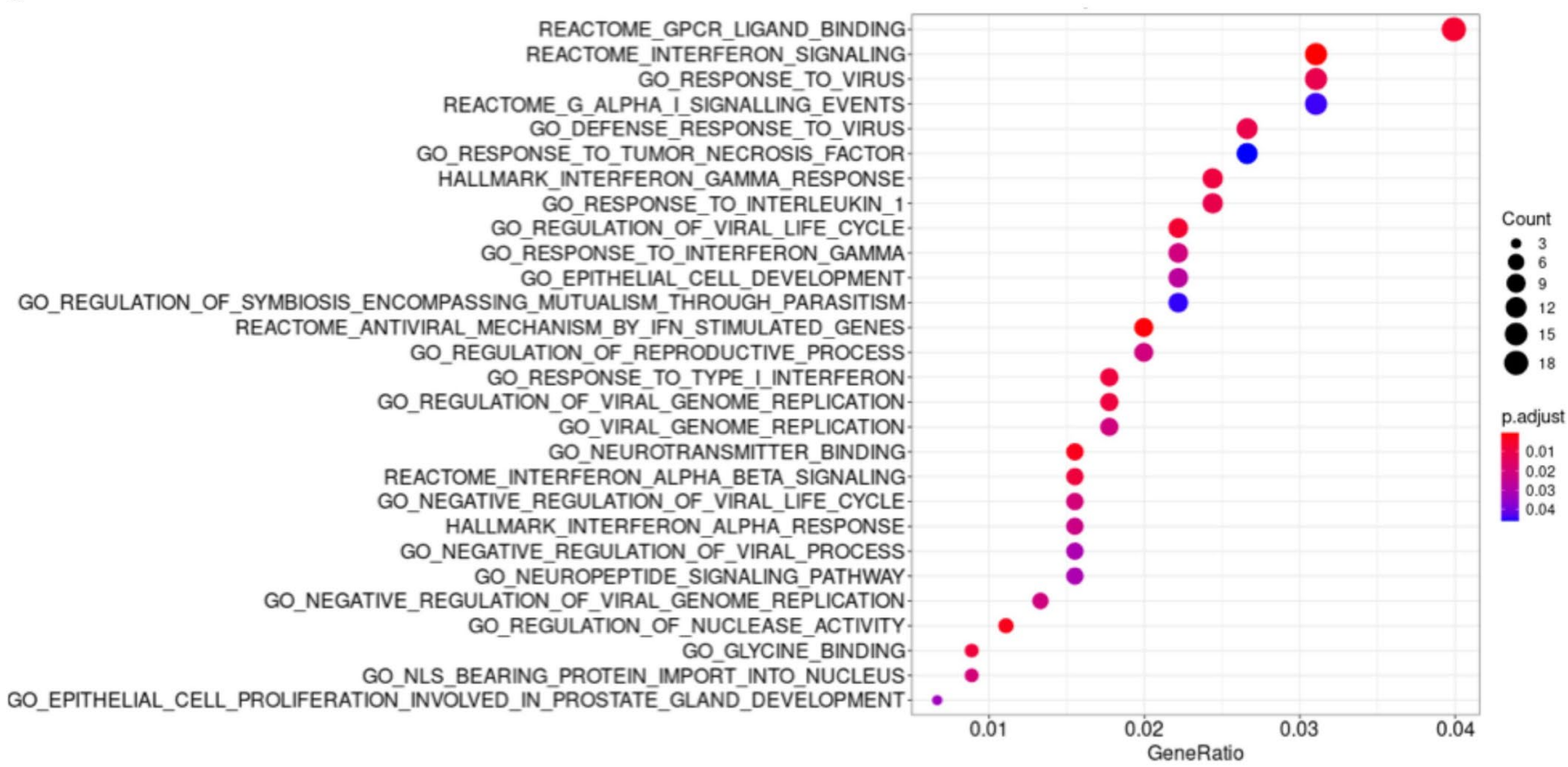

b

REACTOME_SIGNALING_BY_INTERLEUKINS GO_POSITIVE_REGULATION_OF_CYTOKINE_PRODUCTION. GO_COLLAGEN_CONTAINING_EXTRACELLULAR_MATRIX REACTOME_CLASS_IMHC_MEDIATED_ANTIGEN_PROCESSING_PRESENTATION GO_COAGULATION GO_MACROAUTOPHAGY REACTOME_ANTIGEN_PROCESSING_UBIQUITINATION_PROTEASOME_DEGRADATION HALLMARK_HYPOXIA. GO_POSITIVE_REGULATION_OF_CHEMOTAXIS GO_VACUOLE_ORGANIZATION. GO_TOR_SIGNALING. GO_POSITIVE_REGULATION_OF_LEUKOCYTE_CHEMOTAXIS GO_REGULATION_OF_TOR_SIGNALING GO_REGULATION_OF_SYSTEMIC_ARTERIAL_BLOOD_PRESSURE. GO_REGULATION_OF_VIRAL_TRANSCRIPTION. GO_ADHERENS_JUNCTION_ORGANIZATION REACTOME_FORMATION_OF_FIBRIN_CLOT_CLOTTING_CASCADE. GO_NEGATIVE_REGULATION_OF_VIRAL_TRANSCRIPTION. GO_C_C_CHEMOKINE_BINDING.

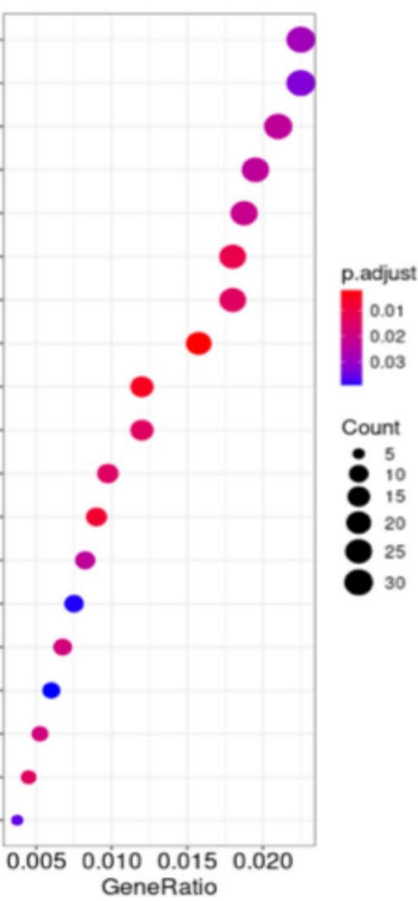

Extended Data Fig. 2 | Enrichment analysis of peak eQTLs for IFNAR2-IL10RB and ACE2 regions. Results obtained from association analysis using all 49 tissues from GTEx V8 contrasted against variant genotypes in an additive model. Dotplot of over-representation analysis using all significant $(p<0.05$ ) differentially expressed (DE) genes (476 for rs13050728; 1,397 for rs4830976) for $\mathbf{a}$, rs13050728, peak eQTL in the IFNAR2-IL10RB region and b, rs4830976, peak eQTL in the ACE2 region. Count = number of DE genes part of the enriched pathway. Gene ratio is the rate of DE genes represented in each pathway. 
a

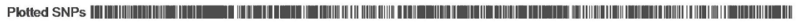

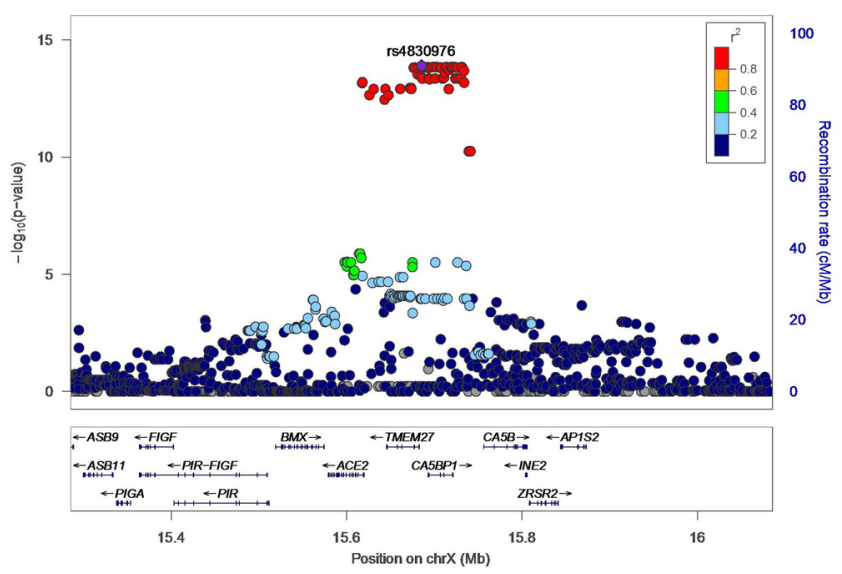

C

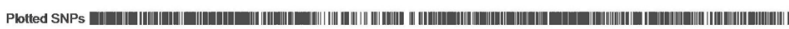

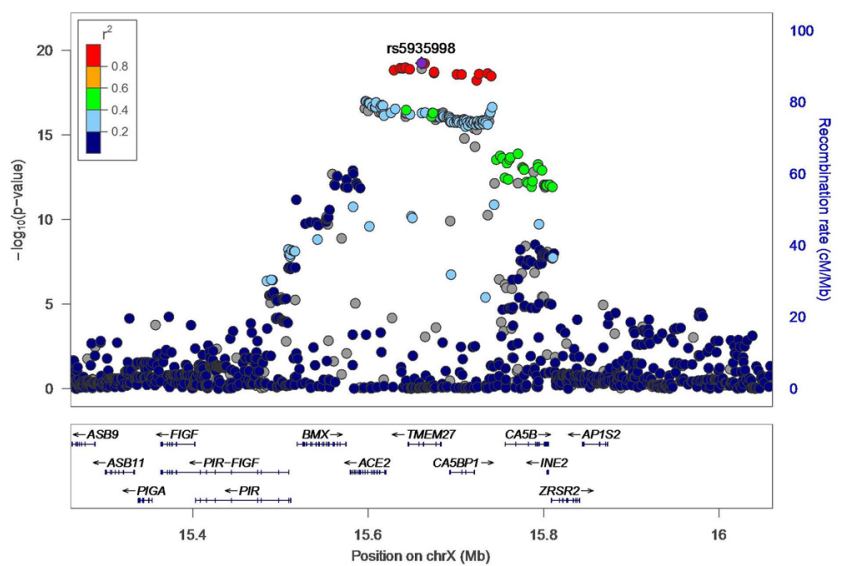

e

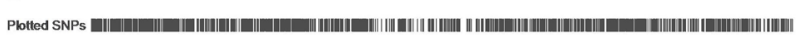

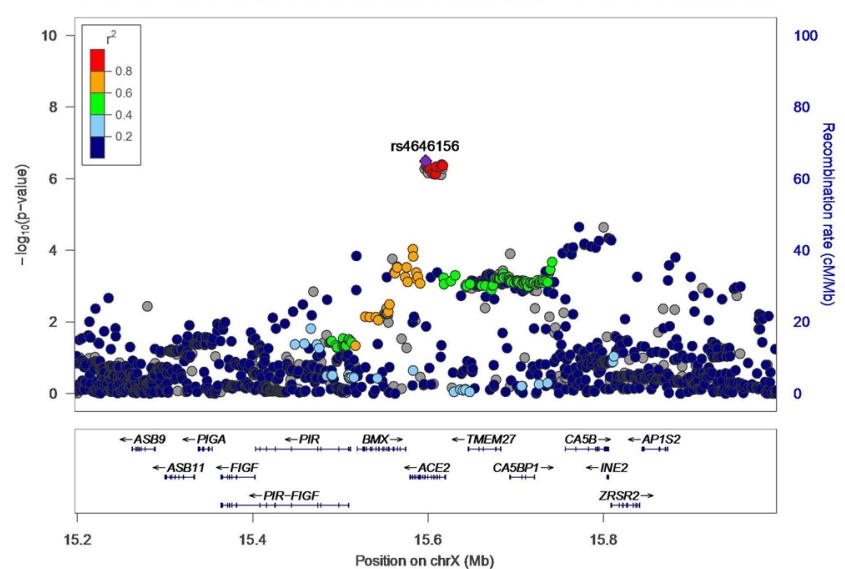

b

Plotted SNPs

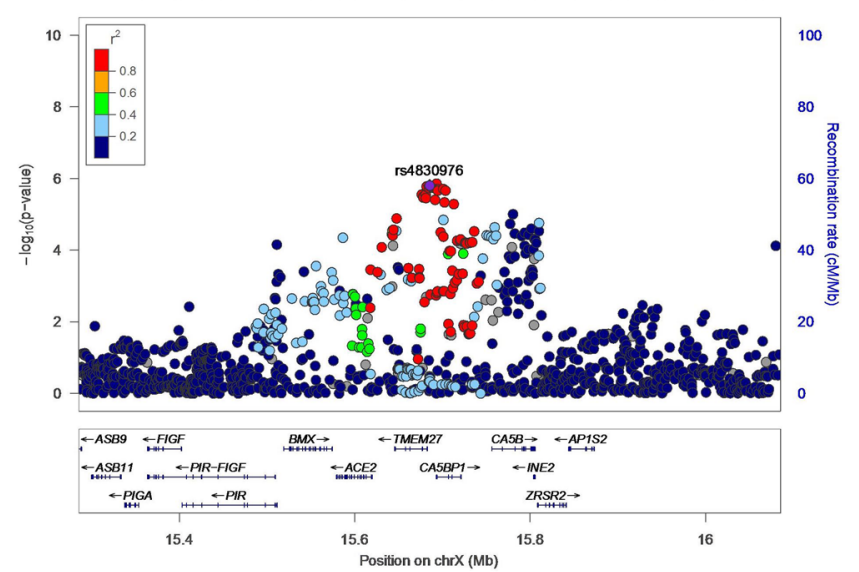

d

Ploted SNPS G|

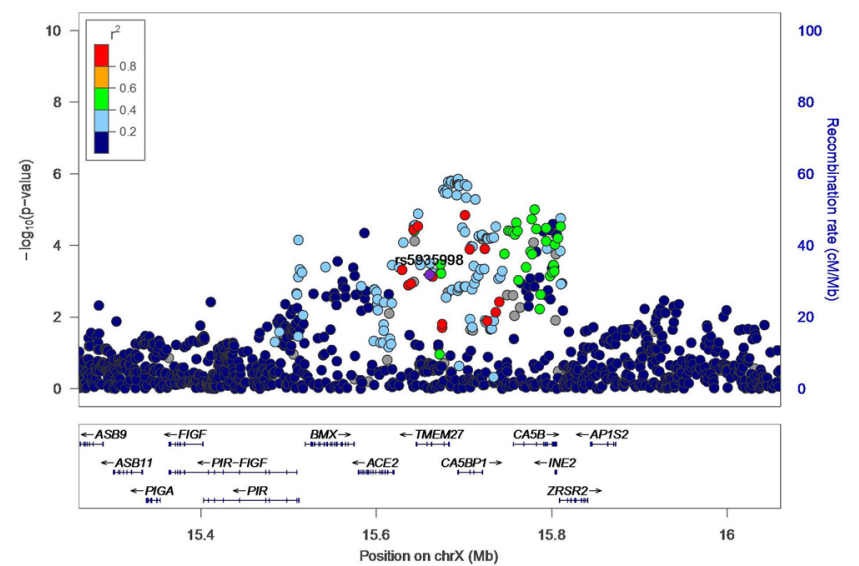

f

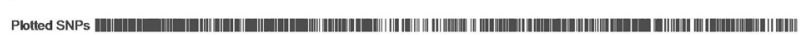

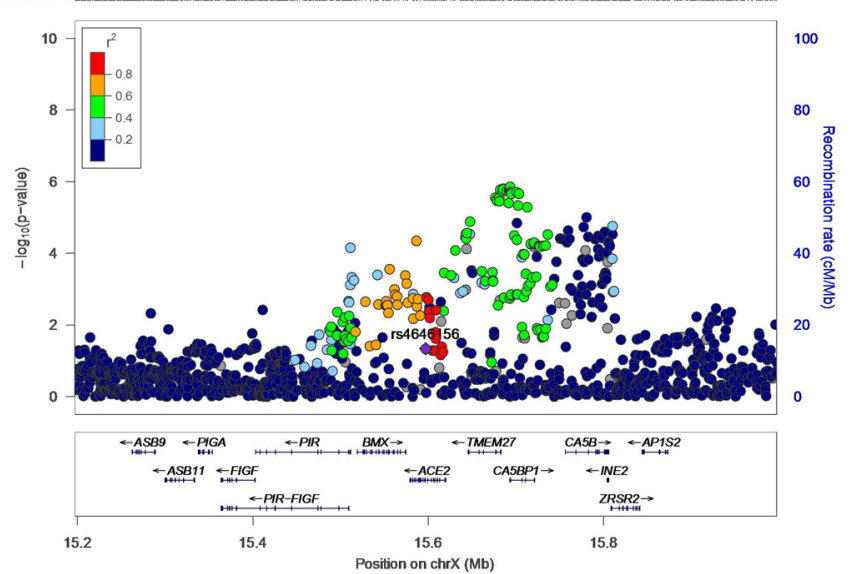

Extended Data Fig. 3 | Regional association plots for cis-variants associated with ACE2 gene expression or ACE2 plasma protein levels, and their association with COVID-19 hospitalization. a, rs4830976 as an eQTL for ACE2 expression in brain frontal cortex tissue (N=175). b, rs4830976 in COVID19 hospitalization $(N=1,377,758)$. c, rs5935998 as the primary pQTL for plasma ACE2 measured by Oink in 4,998 INTERVAL participants d, rs5935998 in COVID-19 hospitalization e, rs4646156 as the secondary PQTL (that is after adjusting for rs5935998) for plasma ACE2 measured by Oink in 4,998 INTERVAL participants. f, rs 4646156 in COVID-19 hospitalization. a colocalizes with b (PP.H4 = 0.95), but c does not colocalize with d (PP.H4 = 0.49$)$ and e does not colocalize with $\mathbf{f}($ PP. $\mathrm{H} 4=0.08)$. 


\section{Reporting Summary}

Nature Research wishes to improve the reproducibility of the work that we publish. This form provides structure for consistency and transparency in reporting. For further information on Nature Research policies, see our Editorial Policies and the Editorial Policy Checklist.

\section{Statistics}

For all statistical analyses, confirm that the following items are present in the figure legend, table legend, main text, or Methods section.

$\mathrm{n} / \mathrm{a}$ Confirmed

$\bigotimes$ The exact sample size $(n)$ for each experimental group/condition, given as a discrete number and unit of measurement

Х $\square$ A statement on whether measurements were taken from distinct samples or whether the same sample was measured repeatedly

The statistical test(s) used AND whether they are one- or two-sided

Only common tests should be described solely by name; describe more complex techniques in the Methods section.

A description of all covariates tested

A description of any assumptions or corrections, such as tests of normality and adjustment for multiple comparisons

A full description of the statistical parameters including central tendency (e.g. means) or other basic estimates (e.g. regression coefficient)

AND variation (e.g. standard deviation) or associated estimates of uncertainty (e.g. confidence intervals)

For null hypothesis testing, the test statistic (e.g. $F, t, r$ ) with confidence intervals, effect sizes, degrees of freedom and $P$ value noted Give $P$ values as exact values whenever suitable.

\For Bayesian analysis, information on the choice of priors and Markov chain Monte Carlo settings

$\bigotimes \square$ For hierarchical and complex designs, identification of the appropriate level for tests and full reporting of outcomes

$\bigotimes \square$ Estimates of effect sizes (e.g. Cohen's $d$, Pearson's $r$ ), indicating how they were calculated

\section{Our web collection on statistics for biologists contains articles on many of the points above.}

\section{Software and code}

Policy information about availability of computer code

Data collection GWAS summary statistics were prepared using freely available software including Matrix eQTL (http://www.bios.unc.edu/research/ genomic_software/Matrix_eQTL/), PLINK v2 (https://www.cog-genomics.org/plink/2.0/), SNPTEST v2.5.2 (https://mathgen.stats.ox.ac.uk/ genetics_software/snptest/snptest.html) and METAL (https://genome.sph.umich.edu/wiki/METAL_Documentation). Software used to prepare imputed genotype data in MVP is described in Supplementary Methods.

Data analysis Analyses were performed in R 3.6.1

Mendelian randomization was performed using the TwoSampleMR R package v0.5.3 (https://mrcieu.github.io/TwoSampleMR/).

Colocalization was performed using the moloc R package (https://github.com/clagiamba/moloc).

Pathway enrichment analysis was performed using the R package clusterProfiler (https://www.ncbi.n/m.nih.gov/pmc/articles/PMC3339379/).

For manuscripts utilizing custom algorithms or software that are central to the research but not yet described in published literature, software must be made available to editors and

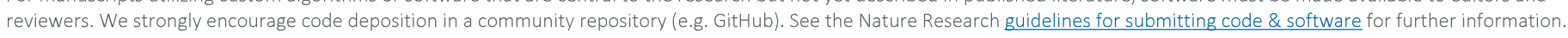

\section{Data}

Policy information about availability of data

All manuscripts must include a data availability statement. This statement should provide the following information, where applicable:

- Accession codes, unique identifiers, or web links for publicly available datasets

- A list of figures that have associated raw data

- A description of any restrictions on data availability

GTEx project version 8 data are available at: https://gtexportal.org/home/. CheMBL database data are available at: https://www.ebi.ac.uk/chembl/. Fenland- 


\section{Field-specific reporting}

Please select the one below that is the best fit for your research. If you are not sure, read the appropriate sections before making your selection.

$\bigotimes$ Life sciences $\quad \square$ Behavioural \& social sciences $\quad \square$ Ecological, evolutionary \& environmental sciences

For a reference copy of the document with all sections, see nature.com/documents/nr-reporting-summary-flat.pdf

\section{Life sciences study design}

All studies must disclose on these points even when the disclosure is negative.

Sample size Instrumental variants were derived from publicly available data ranging from 70 to 10,708 individuals. The outcome GWAS for COVID-19 hospitalization included 7,554 cases and 1,370,204 controls, which was the maximum amount of cases and controls available to us at the time.

Data exclusions Prior to the analysis we decided to restrict instrumental variants to conditionally independent variants that act locally (cis), as this reduces the chances that instrumental variants exhibit horizontal pleiotropy. In MVP, COVID-19 hospitalization cases were restricted to those that occurred between March 1st and September 17, 2020, as this was the data-freeze date at the time we started our analysis.

Replication

For Mendelian randomization (MR) results using eQTL instruments that passed our significance and colocalization thresholds, we tested pQTL instruments derived from plasma proteins measured in INTERVAL participants using an independent platform (Olink), when available. We found that that MR using pQTLs for IL-1ORB did not replicate findings using eQTL (GTEx) instruments, providing evidence that IFNAR2 is perhaps more responsible for the association with COVID-19 hospitalization at that locus. We also found that MR using pQTLS for ACE2 measured by Olink produced a similar effect to MR using eQTL instruments, however pQTL data for ACE2 measured by Olink did not strongly colocalize with the COVID-19 hospitalization summary statistics for the region.

Randomization n/a: This is an observational study and did not randomly allocate individuals to a particular intervention.

Blinding

$\mathrm{n} / \mathrm{a}$ : This is an observational study and did not require blinding to a treatment status.

\section{Reporting for specific materials, systems and methods}

We require information from authors about some types of materials, experimental systems and methods used in many studies. Here, indicate whether each material, system or method listed is relevant to your study. If you are not sure if a list item applies to your research, read the appropriate section before selecting a response.

\begin{tabular}{l|l} 
Materials \& experimental syste \\
\hline$n / a$ & Involved in the study \\
$\searrow$ & $\square$ Antibodies \\
$\searrow$ & $\square$ Eukaryotic cell lines \\
$\searrow$ & $\square$ Palaeontology and archaeology \\
$\searrow$ & $\square$ Animals and other organisms \\
$\square$ & $\square$ Human research participants \\
$\searrow$ & $\square$ Clinical data \\
$\searrow$ & $\square$ Dual use research of concern
\end{tabular}

\begin{tabular}{l|l} 
Methods \\
\hline n/a & Involved in the study \\
$\square$ & $\square$ ChIP-seq \\
$\searrow$ & $\square$ Flow cytometry \\
$\square$ & $\square$ MRI-based neuroimaging
\end{tabular}

\section{Human research participants}

Policy information about studies involving human research participants

Population characteristics

Recruitment
MVP participants ( $n=530,991$ ) were predominantly male (90.4\%), and only European (72.0\%), African American (19.6\%), or Hispanic (8.5\%) ancestries were included (due to insufficient cases in other ancestries). The average age at study enrollment was 62.8 for European, 57.3 for African American, and 54.3 for Hispanic participants. Mean body mass index (BMI) was 29.8 for European, 30.3 for African American, and 30.5 for Hispanic participants. Proportion of male participants was $91.8 \%$ for European, $85.4 \%$ for African American, and $89.5 \%$ for Hispanic participants. The prevalence of COVID-19 hospitalization was $0.32 \%$ for Europeans, $0.61 \%$ for Hispanics, and $0.76 \%$ for African Americans.

Individuals aged 19 to 104 years have been recruited voluntarily from more than 63 VA Medical Centers nationwide for participation in the Million Veteran Program biobank study. Recruitment is currently occurring in person at selected sites in the VHA health care system. Every Veteran is assigned a study ID number, which is used to track them throughout the entire process of recruitment, enrollment, sample collection and use; this approach also provides a level of protection for personal identifiers from the outset. Given that study enrollment is voluntary, biases of this study are similar to those of any mega- 
biobank with voluntary enrollment, including survivorship bias. A complete description of the entire MVP Biobank study including recruitment can be found at PMID: 26441289.

\section{Ethics oversight}

VA Central Institutional Review Board as well as local IRBs at all VA sites recruiting participants

Note that full information on the approval of the study protocol must also be provided in the manuscript. 